\title{
Asymmetric Intermolecular Pauson-Khand Reactions of Unstrained Olefins: the (o- Dimethylamino)phenylsulfinyl Group as an Efficient Chiral Auxiliary
}

\author{
Marta Rodríguez Rivero, Juan Carlos de la Rosa, and Juan Carlos Carretero* \\ Departamento de Química Orgánica, Facultad de Ciencias, Universidad Autónoma de Madrid, Cantoblanco, \\ 28049 Madrid. Spain
}

\section{SUPPORTING INFORMATION}

General. All reagents were obtained from commercial suppliers and were used without further purification except NMO that was crystallized from acetone before using. Reactions were monitored by thin-layer chromatography carried out on 0.25 mm Merck silica gel plates (Merck-60 230-400 mesh). Merck-60 230-400 mesh silica gel was used for flash column chromatography. NMR spectra were recorded on Brucker AC-200 or AC-300 instruments and calibrated using residual undeuterated solvent as internal reference. High resolution mass spectra (HRMS) were determined on a Hewlett-Packard HP-5985 mass spectrometer at $70 \mathrm{eV}$ ionising voltage (EI) or under fast atom bombardment (FAB) conditions. Melting points were determined in open-end capillary tubes on a GallemKamp apparatus. Optical rotations were measured on a Perkin-Elmer 241C polarimeter. High-Performance Liquid Chromatography (HPLC) was conducted on an Agilent 1100 instrument, using Daicel Chiralpak AD and Chiralcel OD columns. High-pressure reactions were conducted under 10 Kbar pressure using a custom-made cylinder-piston type apparatus.

\section{A. SYNTHESIS OF STARTING VINYL SULFOXIDES}
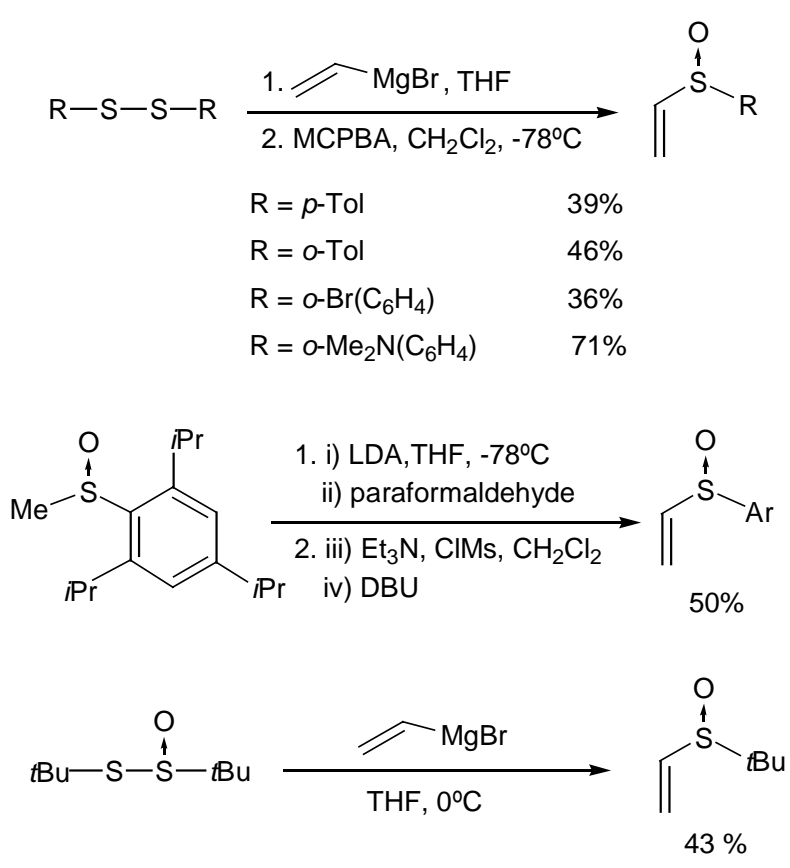
p-Tolyl vinyl sulfoxide (1a): ${ }^{1}$

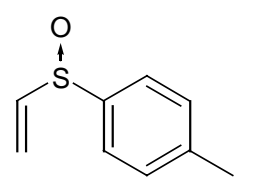

Vinylmagnesium bromide (1M in THF, $1.83 \mathrm{~mL}, 1.83 \mathrm{mmol}$ ) was added to a solution of $p$ tolyl disulfide (300 mg, $1.22 \mathrm{mmol})$ in THF $(10 \mathrm{~mL})$ cooled to $0{ }^{\circ} \mathrm{C}$ under argon. The solution was stirred at $0{ }^{\circ} \mathrm{C}$ for $2 \mathrm{~h}$ and saturated aqueous $\mathrm{NH}_{4} \mathrm{Cl}(10 \mathrm{~mL})$ was then added. The mixture was extracted with $\mathrm{CH}_{2} \mathrm{Cl}_{2}(2 \times 5 \mathrm{~mL})$ and the combined organic layers were dried $\left(\mathrm{Na}_{2} \mathrm{SO}_{4}\right)$ and evaporated. A solution of MCPBA (70\%, $600 \mathrm{mg}, 2.44 \mathrm{mmol})$ in $\mathrm{CH}_{2} \mathrm{Cl}_{2}(15 \mathrm{~mL})$ was added dropwise to a solution of the crude sulfide in $\mathrm{CH}_{2} \mathrm{Cl}_{2}(5 \mathrm{~mL})$ cooled to $-78{ }^{\circ} \mathrm{C}$. After $1 \mathrm{~h}$ at $-78{ }^{\circ} \mathrm{C}$, a saturated solution of $\mathrm{Na}_{2} \mathrm{SO}_{3}(5 \mathrm{~mL})$ followed by a saturated solution of $\mathrm{NaHCO}_{3}(20 \mathrm{~mL})$ were added. The organic layer was separated and the aqueous layer was extracted with $\mathrm{CH}_{2} \mathrm{Cl}_{2}(2 \times 10 \mathrm{~mL})$. The combined organic layers were dried $\left(\mathrm{Na}_{2} \mathrm{SO}_{4}\right)$ and the solvent was evaporated. The residue was purified by flash chromatography (hexane/ethyl acetate $3: 1$ ) to give 1a (80 mg, $39 \%$, colorless oil). ${ }^{1} \mathbf{H}$ NMR (300 MHz, $\left.\mathrm{CDCl}_{3}\right): 7.49$ (d, J=8.1 Hz, 2H, Ar), 7.29 (d, J=8.5 $\mathrm{Hz}, 2 \mathrm{H}, \mathrm{Ar}), 6.56\left(\mathrm{dd}, J=9.3,16.2 \mathrm{~Hz}, 1 \mathrm{H}, \mathrm{C} \underline{\mathrm{H}}=\mathrm{CH}_{2}\right), 6.17\left(\mathrm{~d}, J=16.6 \mathrm{~Hz}, 1 \mathrm{H}, \mathrm{CH}=\mathrm{C}_{2}\right), 5.86(\mathrm{~d}, J=9.7 \mathrm{~Hz}$, $\left.1 \mathrm{H}, \mathrm{CH}=\underline{\mathrm{C}}_{2}\right), 2.38\left(\mathrm{~s}, 3 \mathrm{H}, \mathrm{ArC}_{3}\right) .{ }^{13} \mathrm{C} \mathbf{N M R}\left(75 \mathrm{MHz}, \mathrm{CDCl}_{3}\right): 143.0,141.8,140.0,130.1,124.8,120.3$, 21.4 .

\section{$o$-Tolyl vinyl sulfoxide (1b):}<smiles>C=C[SH](O)c1ccccc1C</smiles>

Vinylmagnesium bromide (1M in THF, $3.65 \mathrm{~mL}, 3.65 \mathrm{mmol}$ ) was added to a solution of $o$-tolyl disulfide $^{2}(600 \mathrm{mg}, 2.44 \mathrm{mmol})$ in THF $(10 \mathrm{~mL})$ cooled to $0{ }^{\circ} \mathrm{C}$ under argon. The solution was stirred at $0{ }^{\circ} \mathrm{C}$ for $2 \mathrm{~h}$ and saturated aqueous $\mathrm{NH}_{4} \mathrm{Cl}(10 \mathrm{~mL})$ was then added. The mixture was extracted with $\mathrm{CH}_{2} \mathrm{Cl}_{2}(2 \times 5 \mathrm{~mL})$ and the combined organic layers were dried $\left(\mathrm{Na}_{2} \mathrm{SO}_{4}\right)$ and evaporated. A solution of MCPBA (70 \%, $1.2 \mathrm{~g}, 4.87 \mathrm{mmol})$ in $\mathrm{CH}_{2} \mathrm{Cl}_{2}(20 \mathrm{~mL})$ was added dropwise to a solution of the crude sulfide in $\mathrm{CH}_{2} \mathrm{Cl}_{2}(10 \mathrm{~mL})$ cooled to $-78^{\circ} \mathrm{C}$. After $1 \mathrm{~h}$ at $-78{ }^{\circ} \mathrm{C}$, a saturated solution of $\mathrm{Na}_{2} \mathrm{SO}_{3}(5 \mathrm{~mL})$ followed by a saturated solution of $\mathrm{NaHCO}_{3}(20 \mathrm{~mL})$ were added. The organic layer was separated and the aqueous layer was extracted with $\mathrm{CH}_{2} \mathrm{Cl}_{2}(2 \times 10 \mathrm{~mL})$. The combined organic layers were dried $\left(\mathrm{Na}_{2} \mathrm{SO}_{4}\right)$ and the solvent was evaporated. The residue was purified by flash chromatography (hexane/ethyl acetate 2:1) to give $\mathbf{1 b}$ (188 $\mathrm{mg}$, $46 \%$, colorless oil). ${ }^{1} \mathbf{H}$ NMR $\left(300 \mathrm{MHz}, \mathrm{CDCl}_{3}\right): 7.79$ (m, 1H, Ar), 7.37 (m, 2H, Ar), 7.20 (m, 1H, Ar), 6.57 (dd, $\left.J=9.2,16.1 \mathrm{~Hz}, 1 \mathrm{H}, \mathrm{C} \underline{\mathrm{H}}=\mathrm{CH}_{2}\right), 6.15\left(\mathrm{~d}, J=16.7 \mathrm{~Hz}, 1 \mathrm{H}, \mathrm{CH}=\underline{\mathrm{C}}_{2}\right), 5.86\left(\mathrm{~d}, J=9.7 \mathrm{~Hz}, 1 \mathrm{H}, \mathrm{CH}=\mathrm{CH}_{2}\right), 2.42$ $\left(\mathrm{s}, 3 \mathrm{H}, \mathrm{ArC}_{3}\right) .{ }^{13} \mathrm{C}$ NMR $\left(75 \mathrm{MHz}, \mathrm{CDCl}_{3}\right): 141.4,135.3,130.9,130.8,127.3,124.0,120.6,18.2$. HRMS (EI+) calcd for $\left(\mathrm{C}_{9} \mathrm{H}_{10} \mathrm{OS}\right)[\mathrm{M}]^{+} 166.0452$, found 166.0458 .

\section{2-Bromophenyl disulfide:}<smiles>Brc1ccccc1SSc1ccccc1Br</smiles>

A solution of 2-bromobenzenethiol $(1.3 \mathrm{~mL}, 10.81 \mathrm{mmol})$ and DMSO $(400 \mu \mathrm{L}, 5.64$ mmol) was stirred at $\mathrm{rt}$ for $24 \mathrm{~h}$. The mixture was cooled to $0{ }^{\circ} \mathrm{C}$, filtered and washed

${ }^{1}$ Mikolajczyk, M.; Perlikowska, W.; Omelanczuk, J.; Cristau, H.-J.; Perraud-Darcy, A. J. Org. Chem. 1998, 63, 97169722.

${ }^{2}$ Karimi, B.; Hazarkhani, H.; Zareyee, D. Synthesis 2002, 2513-2516. 
with cold EtOH to afford 2-bromophenyl disulfide (2.0 g, $95 \%$, white solid). M.p. 99-100 ${ }^{\circ} \mathrm{C}$ [lit. ${ }^{3}$ ? m.p. $93-96$ $\left.{ }^{\circ} \mathrm{C}\right] .{ }^{1} \mathbf{H}$ NMR (200 MHz, $\left.\mathrm{CDCl}_{3}\right): 7.43$ (m, 4H, Ar), 7.27 (dt, J=1.3, 7.3 Hz, 2H, Ar), 7.08 (dt, J=1.6, 7.3 Hz, $2 \mathrm{H}, \mathrm{Ar}) .{ }^{13} \mathbf{C} \mathbf{N M R}\left(50 \mathrm{MHz}, \mathrm{CDCl}_{3}\right): 136.2,132.8,128.2,127.9,127.0,121.1$.

\section{2-Bromophenyl vinyl sulfoxide (1c): ${ }^{4}$}<smiles>C=C[SH](O)c1ccccc1Br</smiles>

Vinylmagnesium bromide (1M in THF, $4.00 \mathrm{~mL}, 3.99 \mathrm{mmol})$ was added to a solution of 2bromophenyl disulfide $(1.00 \mathrm{~g}, 2.66 \mathrm{mmol})$ in THF $(30 \mathrm{~mL})$ cooled to $-78{ }^{\circ} \mathrm{C}$ under argon. The solution was stirred at $-78^{\circ} \mathrm{C}$ for $1 \mathrm{~h}$ and saturated aqueous $\mathrm{NH}_{4} \mathrm{Cl}(25 \mathrm{~mL})$ was then added. The mixture was extracted with $\mathrm{CH}_{2} \mathrm{Cl}_{2}(2 \times 15 \mathrm{~mL})$ and the combined organic layers were dried $\left(\mathrm{Na}_{2} \mathrm{SO}_{4}\right)$ and evaporated. A solution of MCPBA (70\%, $775 \mathrm{mg}, 3.5 \mathrm{mmol})$ in $\mathrm{CH}_{2} \mathrm{Cl}_{2}(20 \mathrm{~mL})$ was added dropwise to a solution of the crude sulfide in $\mathrm{CH}_{2} \mathrm{Cl}_{2}(15 \mathrm{~mL})$ cooled to $-78{ }^{\circ} \mathrm{C}$. After $1 \mathrm{~h}$ at $-78{ }^{\circ} \mathrm{C}$, a saturated solution of $\mathrm{Na}_{2} \mathrm{SO}_{3}(10 \mathrm{~mL})$ followed by a saturated solution of $\mathrm{NaHCO}_{3}(30 \mathrm{~mL})$ were added. The organic layer was separated and the aqueous layer was extracted with $\mathrm{CH}_{2} \mathrm{Cl}_{2}(2 \times 20 \mathrm{~mL})$. The combined organic layers were dried $\left(\mathrm{Na}_{2} \mathrm{SO}_{4}\right)$ and the solvent was evaporated. The residue was purified by flash chromatography (hexane/ethyl acetate 5:2) to give 1c (220 mg, $36 \%$, colorless oil). ${ }^{1} \mathbf{H}$ NMR (200 MHz, $\left.\mathrm{CDCl}_{3}\right): 7.79$ (dd, $J=1.6,7.5 \mathrm{~Hz}, 1 \mathrm{H}, \mathrm{Ar}), 7.53$ (m, 2H, Ar), 7.34 (dt, J=1.6, $7.5 \mathrm{~Hz}, 1 \mathrm{H}, \mathrm{Ar}), 6.88$ (dd, J=9.7, $16.7 \mathrm{~Hz}, 1 \mathrm{H}$, $\left.\mathrm{C} \underline{\mathrm{H}}=\mathrm{CH}_{2}\right), 6.23\left(\mathrm{~d}, J=16.7 \mathrm{~Hz}, 1 \mathrm{H}, \mathrm{CH}=\underline{\mathrm{C}}_{2}\right), 5.89\left(\mathrm{~d}, J=9.1 \mathrm{~Hz}, 1 \mathrm{H}, \mathrm{CH}=\underline{\mathrm{C}}_{2}\right) .{ }^{13} \mathbf{C} \mathbf{N M R}\left(75 \mathrm{MHz}, \mathrm{CDCl}_{3}\right)$ : $143.2,140.3,132.8,132.1,128.6,125.4,120.8,118.8$.

\section{2,4,6-Triisopropylphenyl vinyl sulfoxide $(1 \mathrm{~d}):^{5}$}

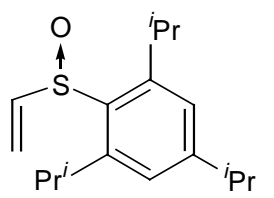

A solution of methyl 2,4,6-triisopropylphenyl sulfoxide (400 mg, $1.50 \mathrm{mmol})$ in THF (10 $\mathrm{mL}$ ) at $-78^{\circ} \mathrm{C}$ under argon, was treated with LDA ( $3.3 \mathrm{~mL}, 1.65 \mathrm{mmol}, 0.5 \mathrm{M}$ in THF), stirred for $30 \mathrm{~min}$ at this temperature, and treated dropwise with a suspension of paraformaldehyde $(135 \mathrm{mg}, 4.50 \mathrm{mmol})$ in THF $(5 \mathrm{~mL})$. After being stirred for $2 \mathrm{~h}$ at $-78{ }^{\circ} \mathrm{C}$, saturated aqueous solution of $\mathrm{NH}_{4} \mathrm{Cl}(10 \mathrm{~mL})$ was added. The mixture was extracted with $\mathrm{CH}_{2} \mathrm{Cl}_{2}(3 \times 10 \mathrm{~mL})$ and the combined organic layers were dried $\left(\mathrm{Na}_{2} \mathrm{SO}_{4}\right)$ and evaporated. To a solution of the resulting alcohol in $\operatorname{dry} \mathrm{CH}_{2} \mathrm{Cl}_{2}(10$ $\mathrm{mL})$ at $0{ }^{\circ} \mathrm{C}$ were added $\mathrm{Et}_{3} \mathrm{~N}(2.10 \mathrm{~mL}, 15.0 \mathrm{mmol})$ and mesyl chloride $(174 \mu \mathrm{l}, 2.25 \mathrm{mmol})$, warmed and stirred for $1 \mathrm{~h}$ at $\mathrm{rt}$, and treated with DBU $(1.12 \mathrm{~mL}, 7.51 \mathrm{mmol})$. After being stirred overnight at $\mathrm{rt}$, the reaction mixture was quenched with saturated aqueous solution of $\mathrm{NH}_{4} \mathrm{Cl}(10 \mathrm{~mL})$ and extracted with $\mathrm{CH}_{2} \mathrm{Cl}_{2}(2 \times 10$ $\mathrm{mL})$. The combined organic layers were dried $\left(\mathrm{Na}_{2} \mathrm{SO}_{4}\right)$ and the solvent was evaporated. The residue was purified by flash chromatography (hexane/ethyl acetate 3:1) to afford $1 \mathbf{1 d}(210 \mathrm{mg}, 50 \%$, white solid). M.p. 76$78{ }^{\circ} \mathrm{C}$ [lit. ${ }^{4}:$ m.p. $70-72{ }^{\circ} \mathrm{C}$ ]. ${ }^{1} \mathbf{H}$ NMR $\left(300 \mathrm{MHz}, \mathrm{CDCl}_{3}\right.$ ): 7.04 (s, 2H, Ar), 6.70 (dd, J=10.5, 16.6 Hz, 1H, $\left.\mathrm{C} \underline{\mathrm{H}}=\mathrm{CH}_{2}\right), 6.06\left(\mathrm{~d}, J=16.2 \mathrm{~Hz}, 1 \mathrm{H}, \mathrm{CH}=\underline{\mathrm{H}}_{2}\right), 5.91\left(\mathrm{~d}, J=10.1 \mathrm{~Hz}, 1 \mathrm{H}, \mathrm{CH}=\underline{\mathrm{C}}_{2}\right), 3.84$ (quint, $J=6.9 \mathrm{~Hz}, 2 \mathrm{H}$,

\footnotetext{
${ }^{3}$ Villemin, D.; Goussu, D. Heterocycles 1989, 29, 1255-1261.

${ }^{4}$ Imboden, C.; Renaud, P. Tetrahedron: Asymmetry 1999, 10, 1051-1060.

${ }^{5}$ Delouvrie, B.; Fensterbank, L.; Lacote, E.; Malacria, M. J. Am. Chem. Soc. 1999, 121, 11395-11401.
} 
$\left.\mathrm{C} \underline{\mathrm{H}}\left(\mathrm{CH}_{3}\right)_{2}\right), 2.85$ (quint, $\left.J=6.9 \mathrm{~Hz}, 1 \mathrm{H}, \mathrm{C} \underline{\mathrm{H}}\left(\mathrm{CH}_{3}\right)_{2}\right), 1.20\left(\mathrm{~m}, 18 \mathrm{H}, \mathrm{CH}\left(\mathrm{C}_{3}\right)_{2}\right) .{ }^{13} \mathbf{C ~ N M R}\left(75 \mathrm{MHz}, \mathrm{CDCl}_{3}\right)$ : $152.8,150.6,142.2,132.8,123.0,119.4,34.2,28.1,24.8,23.6$.

\section{tert-Butyl vinyl sulfoxide (1e): ${ }^{6}$}

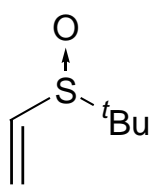

Vinylmagnesium bromide (1M in THF, $3.10 \mathrm{~mL}, 3.10 \mathrm{mmol}$ ) was added to a solution of tert-butyl tert-butanethiosulfinate $(300 \mathrm{mg}, 1.55 \mathrm{mmol})$ in THF $(10 \mathrm{~mL})$ cooled to $0{ }^{\circ} \mathrm{C}$ under argon. The solution was stirred at $0{ }^{\circ} \mathrm{C}$ for $1 \mathrm{~h}$ and saturated aqueous $\mathrm{NH}_{4} \mathrm{Cl}(10 \mathrm{~mL})$ was then added. The mixture was extracted with $\mathrm{CH}_{2} \mathrm{Cl}_{2}(2 \times 15 \mathrm{~mL})$ and the combined organic layers were dried $\left(\mathrm{Na}_{2} \mathrm{SO}_{4}\right)$ and evaporated. The residue was purified by flash chromatography (hexane/ethyl acetate 1:1) to give $1 \mathbf{e}$ (87 $\mathrm{mg}, 43$ \%, colorless oil). ${ }^{1} \mathbf{H}$ NMR $\left(200 \mathrm{MHz}, \mathrm{CDCl}_{3}\right): 6.55$ (dd, $J=10.2,16.7 \mathrm{~Hz}, 1 \mathrm{H}, \mathrm{C} \underline{\mathrm{H}}=\mathrm{CH}_{2}$ ), 6.07 (d, $J=16.7 \mathrm{~Hz}$, $\left.1 \mathrm{H}, \mathrm{CH}=\mathrm{CH}_{2}\right), 6.01\left(\mathrm{~d}, J=9.7 \mathrm{~Hz}, 1 \mathrm{H}, \mathrm{CH}=\mathrm{CH}_{2}\right), 1.22(\mathrm{~s}, 9 \mathrm{H}, t-\mathrm{Bu}) .{ }^{13} \mathbf{C} \mathbf{N M R}\left(50 \mathrm{MHz}, \mathrm{CDCl}_{3}\right): 136.4,123.5$, 54.5, 22.6.

\section{$\operatorname{Bis}[2-(N, N$-dimethylamino)phenyl] disulfide:}<smiles>CN(C)c1ccccc1SSc1ccccc1N</smiles>
MeI $(1.0 \mathrm{~mL}, 16.0 \mathrm{mmol})$ was added to a solution of 2-aminophenyl disulfide $7.0 \mathrm{~g}$, $4.0 \mathrm{mmol})$ and $\mathrm{K}_{2} \mathrm{CO}_{3}(2.2 \mathrm{~g}, 16.0 \mathrm{mmol})$ in $\mathrm{CH}_{3} \mathrm{CN}(80 \mathrm{~mL})$, under argon, at rt. The mixture was stirred at $\mathrm{rt}$ for 4 days and $\mathrm{H}_{2} \mathrm{O}(20 \mathrm{~mL})$ was then added. The mixture was extracted with $\mathrm{CH}_{2} \mathrm{Cl}_{2}(3 \times 15 \mathrm{~mL})$ and the combined organic layers were dried $\left(\mathrm{Na}_{2} \mathrm{SO}_{4}\right)$ and evaporated. The residue was purified by flash chromatography (hexane/ethyl acetate 6:1) to give bis[2-(N,Ndimethylamino)phenyl] disulfide (1.0 g, $90 \%$, yellow solid). M.p. $81-82{ }^{\circ} \mathrm{C} .{ }^{1} \mathbf{H}$ NMR $\left(300 \mathrm{MHz}, \mathrm{CDCl}_{3}\right): 7.48$ (dd, $J=1.6,8.6 \mathrm{~Hz}, 2 \mathrm{H}, \mathrm{Ar}), 7.18-7.12$ (m, 4H, Ar), 7.08-6.98(m, 2H, Ar), 2.83 (s, 12H, N( $\left.\left.\mathrm{CH}_{3}\right)_{2}\right) .{ }^{13} \mathbf{C} \mathbf{N M R}$ (75 MHz, $\left.\mathrm{CDCl}_{3}\right): 151.4,132.3,126.5,125.5,124.7,119.8,44.9$.

\section{2-(N,N-Dimethylamino)phenyl vinyl sulfoxide (1f):}<smiles>C=CO[SH](O)c1ccccc1N(C)C</smiles>

Vinylmagnesium bromide (1M in THF, $4.90 \mathrm{~mL}, 4.90 \mathrm{mmol})$ was added to a solution of 2$(N, N$-dimethylamino $)$ phenyl disulfide $(1.00 \mathrm{~g}, 3.8 \mathrm{mmol})$ in THF $(30 \mathrm{~mL})$ cooled to $0{ }^{\circ} \mathrm{C}$ under argon. The solution was stirred at $\mathrm{rt}$ for $1 \mathrm{~h}$ and saturated aqueous $\mathrm{NH}_{4} \mathrm{Cl}(25 \mathrm{~mL})$ was then added. The mixture was extracted with $\mathrm{CH}_{2} \mathrm{Cl}_{2}(2 \times 15 \mathrm{~mL})$ and the combined organic layers were dried $\left(\mathrm{Na}_{2} \mathrm{SO}_{4}\right)$ and evaporated. A solution of MCPBA $(70 \%, 1.62 \mathrm{~g}, 6.57 \mathrm{mmol})$ in $\mathrm{CH}_{2} \mathrm{Cl}_{2}(40 \mathrm{~mL})$ was added dropwise to a solution of the crude sulfide in $\mathrm{CH}_{2} \mathrm{Cl}_{2}(15 \mathrm{~mL})$ cooled to $-78{ }^{\circ} \mathrm{C}$. After $1 \mathrm{~h}$ at $-78{ }^{\circ} \mathrm{C}$, a saturated solution of $\mathrm{Na}_{2} \mathrm{SO}_{3}(10 \mathrm{~mL})$ followed by a saturated solution of $\mathrm{NaHCO}_{3}(30 \mathrm{~mL})$ were added. The organic layer was separated and the aqueous layer was extracted with $\mathrm{CH}_{2} \mathrm{Cl}_{2}(2 \times 20 \mathrm{~mL})$. The combined organic layers were dried $\left(\mathrm{Na}_{2} \mathrm{SO}_{4}\right)$ and the solvent was evaporated. The residue was purified by flash chromatography (hexane/ethyl acetate 4:1) to afford $\mathbf{1 f}\left(452 \mathrm{mg}, 71 \%\right.$, colorless oil). ${ }^{1} \mathbf{H} \mathbf{N M R}\left(200 \mathrm{MHz}, \mathrm{CDCl}_{3}\right): 7.74$ (dd,

\footnotetext{
${ }^{6}$ Cogan, D. A.; Liu, G.; Kim, K.; Backes, B. J.; Ellman, J. A. J. Am. Chem. Soc. 1998, 120, 8011-8019.

${ }^{7}$ Fristad, W. E.; Peterson, J. R. Synthetic Communications 1985, 15, 1-5.
} 
$J=1.6,7.5 \mathrm{~Hz}, 1 \mathrm{H}, \mathrm{Ar}), 7.42$ (dt, J=1.6, 7.5 Hz, 1H, Ar), 7.26 (dt, J=1.1, 7.5 Hz, 1H, Ar), 7.17 (dd, J=1.1, 7.5 $\mathrm{Hz}, 1 \mathrm{H}, \mathrm{Ar}), 6.95$ (dd, J=9.7, $\left.16.1 \mathrm{~Hz}, 1 \mathrm{H}, \mathrm{C} \underline{\mathrm{H}}=\mathrm{CH}_{2}\right), 6.10\left(\mathrm{~d}, J=16.7 \mathrm{~Hz}, 1 \mathrm{H}, \mathrm{CH}=\underline{\mathrm{C}}_{2}\right), 5.79$ (d, J=9.1 Hz, $\left.1 \mathrm{H}, \mathrm{CH}=\mathrm{CH}_{2}\right), 2.76\left(\mathrm{~s}, 6 \mathrm{H}, \mathrm{N}\left(\mathrm{CH}_{3}\right)_{2}\right) .{ }^{13} \mathbf{C}$ NMR $\left(50 \mathrm{MHz}, \mathrm{CDCl}_{3}\right): 150.4,141.3,137.8,131.0,124.6,123.4$, 119.8, 118.1, 44.5. HRMS (EI+) calcd for $\left(\mathrm{C}_{10} \mathrm{H}_{13} \mathrm{NOS}\right)[\mathrm{M}]^{+}$195.0718, found 195.0724.

\section{B. PAUSON-KHAND REACTION}

2-n-Butyl- 5-(p-tolylsulfinyl)-2-cyclopentenone (2a):

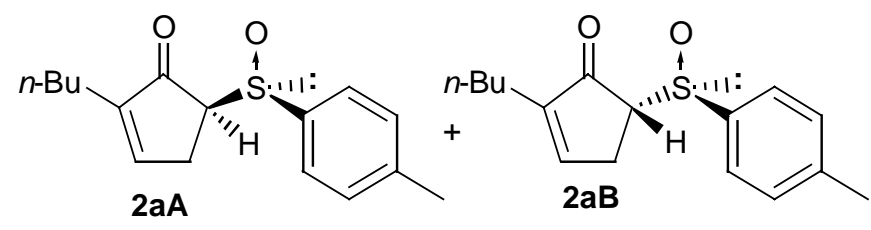

A solution of 1-hexyne $(26 \mu \mathrm{l}, 0.23 \mathrm{mmol})$ and $\mathrm{Co}_{2}(\mathrm{CO})_{8}(77 \mathrm{mg}, 0.23 \mathrm{mmol})$ in $\mathrm{CH}_{3} \mathrm{CN}(1 \mathrm{~mL})$ was stirred under argon atmosphere at rt. After $1 \mathrm{~h}$

$N$-methylmorpholine $N$-oxide (106 mg, $0.90 \mathrm{mmol}$ )

and a solution of $1 \mathbf{a}(25 \mathrm{mg}, 0.15 \mathrm{mmol})$ in $\mathrm{CH}_{3} \mathrm{CN}(1 \mathrm{~mL})$ were added and the resulting solution was stirred for $2 \mathrm{~h}$. The reaction mixture was diluted with $\mathrm{CH}_{2} \mathrm{Cl}_{2}$, treated with trimethyl amine $N$-oxide (50 mg) to remove the remaining cobalt complex and stirred for $10 \mathrm{~min}$ at $\mathrm{rt}$. After removal of the solvent, the crude product was dissolved in diethyl ether and filtered trough a pad of Celite. The combined organic solvents were evaporated and the residue was purified by chromatography (hexane/ethyl acetate 3:1) to afford $\mathbf{2} \mathbf{a A}$ and $\mathbf{2} \mathbf{a B}$ (28 $\mathbf{m g}, 68$ $\%$, colorless oil) as a 74:26 diastereomeric mixture.

A : ${ }^{1} \mathbf{H}$ NMR $\left(300 \mathrm{MHz}, \mathrm{CDCl}_{3}\right): 7.52-7.21(\mathrm{~m}, 5 \mathrm{H}, \mathrm{Ar}, \mathrm{H}-3), 3.49$ (dd, J=2.2, $\left.6.5 \mathrm{~Hz}, 1 \mathrm{H}, \mathrm{H}-5\right), 3.04-2.89$ (m, 1H, H-4), 2.41 (s, 3H, $\mathrm{ArCH}_{3}$ ), 2.40-2.26 (m, 1H, H-4), 2.18 (m, 2H, H-1'), 1.51-1.09, (m, 4H, H-2', H-3'), 0.88 (t, J=7.0 Hz, 3H, H-4'). ${ }^{13} \mathbf{C}$ NMR (75 MHz, $\mathrm{CDCl}_{3}$ ): 201.7, 201.0, 157.7, 156.9, 147.0, 146.8, 142.1, 141.6, 139.1, 135.4, 129.9, 129.5, 125.0, 124.0, 68.1, 66.0, 29.6, 29.5, 29.3, 25.5, 24.7, 24.2, 23.9, 22.3, 22.1, 21.4, 13.8, 13.7. HRMS (FAB+) calcd for $\left(\mathrm{C}_{16} \mathrm{H}_{20} \mathrm{O}_{2} \mathrm{~S}\right)[\mathrm{M}]^{+} 276.1184$, found 276.1177.

B: ${ }^{1} \mathbf{H}$ NMR (300 MHz, $\mathrm{CDCl}_{3}$ ) (significant signals): $7.03(\mathrm{~m}, 1 \mathrm{H}, \mathrm{H}-3), 4.20$ (dd, J=2.2, $\left.6.5 \mathrm{~Hz}, 1 \mathrm{H}, \mathrm{H}-5\right)$, 2.85-2.70 (m, 1H, H-4), 2.36 (s, 3H, $\left.\mathrm{ArC}_{3}\right), 1.91$ (m, 2H, H-1').

\section{2-n-Butyl- 5-(o-tolylsulfinyl)-2-cyclopentenone (2b):}

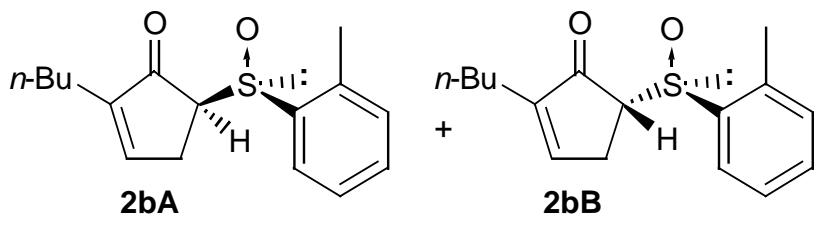

A solution of 1-hexyne $(52 \mu \mathrm{l}, 0.45 \mathrm{mmol})$ and $\mathrm{Co}_{2}(\mathrm{CO})_{8}(154 \mathrm{mg}, 0.45 \mathrm{mmol})$ in $\mathrm{CH}_{3} \mathrm{CN}(2 \mathrm{~mL})$ was stirred under argon atmosphere at rt. After $1 \mathrm{~h} \mathrm{~N}$ methylmorpholine $N$-oxide $(211 \mathrm{mg}, 1.80 \mathrm{mmol})$ and a

solution of $1 \mathbf{b}(50 \mathrm{mg}, 0.30 \mathrm{mmol})$ in $\mathrm{CH}_{3} \mathrm{CN}(2 \mathrm{~mL})$ were added. After $10 \mathrm{~h}$ at $\mathrm{rt}$ another 1.5 equivalents of the cobalt complex were added and the resulting solution was stirred for $14 \mathrm{~h}$ at $\mathrm{rt}$. The reaction mixture was diluted with $\mathrm{CH}_{2} \mathrm{Cl}_{2}$, treated with trimethyl amine $\mathrm{N}$-oxide $(200 \mathrm{mg}$ ) and stirred for $10 \mathrm{~min}$ at $\mathrm{rt}$. After removal of the solvent, the crude product was dissolved in diethyl ether and filtered trough a pad of Celite. The combined organic solvents were evaporated and the residue was purified by chromatography (hexane/ethyl acetate 5:1) to afford $\mathbf{2 b A}$ and $\mathbf{2 b B}$ (23 $\mathrm{mg}, 28 \%$, colorless oil) as a 90:10 diastereomeric mixture. 
A: ${ }^{1} \mathbf{H}$ NMR $\left(300 \mathrm{MHz}, \mathrm{CDCl}_{3}\right): 7.82(\mathrm{dd}, J=2.8,7.3 \mathrm{~Hz}, 1 \mathrm{H}, \mathrm{Ar}), 7.44-7.35$ (m, 3H, Ar, H-3), 7.21 (dd, $J=2.4$, $6.1 \mathrm{~Hz}, 1 \mathrm{H}, \mathrm{Ar}), 3.53$ (dd, J=2.4, $6.9 \mathrm{~Hz}, 1 \mathrm{H}, \mathrm{H}-5), 3.06-2.97$ (m, 1H, H-4), 2.42 (s, 3H, $\mathrm{ArCH}_{3}$ ), 2.33-2.17 (m, 3H, H-4, H-1'), 1.51-1.23, (m, 4H, H-2', H-3'), 0.89 (t, J=7.3 Hz, 3H, H-4'). ${ }^{13} \mathbf{C}$ NMR (75 MHz, CDCl $)_{3}$ ): 202.0, 158.0, 155.9, 146.9, 140.4, 134.2, 130.9, 126.9, 124.1, 65.0, 29.5, 24.7, 23.2, 22.3, 18.0, 13.7. HRMS (EI+) calcd for $\left(\mathrm{C}_{16} \mathrm{H}_{20} \mathrm{O}_{2} \mathrm{~S}\right)[\mathrm{M}]^{+} 276.1184$, found 276.1182 .

B: ${ }^{1} \mathbf{H}$ NMR (300 MHz, $\mathrm{CDCl}_{3}$ ) (significant signals): 3.94 (dd, J=1.6, $\left.6.9 \mathrm{~Hz}, 1 \mathrm{H}, \mathrm{H}-5\right), 2.36$ (s, 3H, $\left.\mathrm{ArCH}_{3}\right)$.

2-n-Butyl- 5-(2-bromophenylsulfinyl)-2-cyclopentenone (2c):

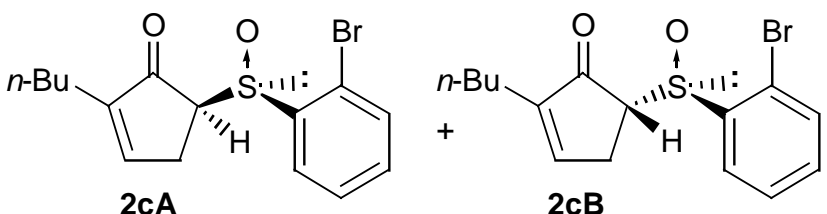

A solution of 1-hexyne $(22 \mu \mathrm{l}, 0.19 \mathrm{mmol})$ and $\mathrm{Co}_{2}(\mathrm{CO})_{8}(67 \mathrm{mg}, 0.19 \mathrm{mmol})$ in $\mathrm{CH}_{3} \mathrm{CN}(1 \mathrm{~mL})$ was stirred under argon atmosphere at rt. After $1 \mathrm{~h} \mathrm{~N}$ methylmorpholine $\mathrm{N}$-oxide $(91 \mathrm{mg}, 0.78 \mathrm{mmol})$ and a

solution of $1 \mathbf{c}$ (30 mg, $0.13 \mathrm{mmol})$ in $\mathrm{CH}_{3} \mathrm{CN}(1 \mathrm{~mL})$ were added. After $12 \mathrm{~h}$ at $\mathrm{rt}$ another 1.5 equivalents of the cobalt complex were added and the resulting solution was stirred for $12 \mathrm{~h}$ at rt. The reaction mixture was diluted with $\mathrm{CH}_{2} \mathrm{Cl}_{2}$, treated with trimethyl amine $\mathrm{N}$-oxide $(90 \mathrm{mg}$ ) and stirred for $10 \mathrm{~min}$ at rt. After removal of the solvent, the crude product was dissolved in diethyl ether and filtered trough a pad of Celite. The combined organic solvents were evaporated and the residue was purified by chromatography (hexane/ethyl acetate $3: 1$ ) to afford $\mathbf{2 c A}$ and $\mathbf{2 c B}$ (15 mg, $30 \%$, colorless oil) as an 86:14 diastereomeric mixture.

A : ${ }^{1} \mathbf{H}$ NMR (300 MHz, CDCl $)$ ): $7.82(\mathrm{dd}, J=1.6,7.5 \mathrm{~Hz}, 1 \mathrm{H}, \mathrm{Ar}), 7.57$ (t, J=7.5 Hz, 2H, Ar), 7.39 (m, 2H, H-3, Ar), 4.09 (dd, J=2.7, 7.0 Hz, 1H, H-5), 2.89 (m, 1H, H-4), 2.21 (m, 3H, H-1', H-4), 1.56-1.21 (m, 4H, H-2', H3'), 0.90 (t, J=7.0 Hz, 3H, H-4'). ${ }^{13} \mathbf{C}$ NMR (75 MHz, $\mathrm{CDCl}_{3}$ ): 201.7, 157.7, 147.1, 141.6, 133.2, 132.5, 128.3, 127.1, 118.7, 63.9, 29.5, 24.8, 23.3, 22.4, 13.8. HRMS (EI+) calcd for $\left(\mathrm{C}_{15} \mathrm{H}_{17} \mathrm{O}_{2} \mathrm{SBr}\right)[\mathrm{M}]^{+} 340.0133$, found 340.0132 .

B: ${ }^{1} \mathbf{H}$ NMR (300 MHz, CDCl $)$ (significant signals): 3.97 (m, 1H, H-5), 3.19 (m, 1H, H-4).

\section{2-n-Butyl- 5-(2,4,6-triisopropylphenylsulfinyl)-2-cyclopentenone (2d):}

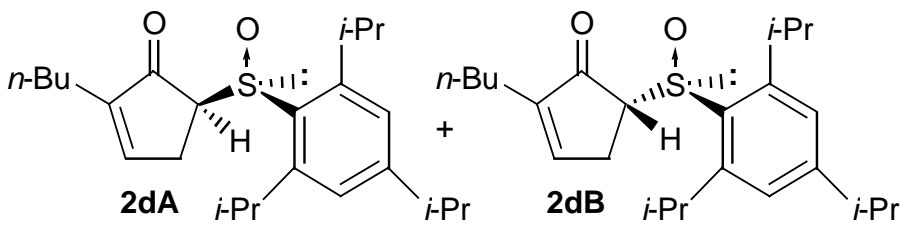

A solution of 1-hexyne $(19 \mu \mathrm{l}, 0.16 \mathrm{mmol})$ and $\mathrm{Co}_{2}(\mathrm{CO})_{8}(55 \mathrm{mg}, 0.16 \mathrm{mmol})$ in $\mathrm{CH}_{3} \mathrm{CN}(1 \mathrm{~mL})$ was stirred under argon atmosphere at rt. After $1 \mathrm{~h}$ $N$-methylmorpholine $N$-oxide (76 mg, $0.65 \mathrm{mmol}$ )

and a solution of $\mathbf{1 d}(30 \mathrm{mg}, 0.11 \mathrm{mmol})$ in $\mathrm{CH}_{3} \mathrm{CN}(1 \mathrm{~mL})$ were added. After $12 \mathrm{~h}$ at rt another 1.5 equivalents of the cobalt complex were added and the resulting solution was stirred for $12 \mathrm{~h}$. The reaction mixture was diluted with $\mathrm{CH}_{2} \mathrm{Cl}_{2}$, treated with trimethyl amine $\mathrm{N}$-oxide $(70 \mathrm{mg}$ ) and stirred for $10 \mathrm{~min}$ at rt. After removal of the solvent, the crude product was dissolved in diethyl ether and filtered trough a pad of Celite. The combined organic solvents were evaporated and the residue was purified by chromatography (hexane/ethyl acetate $5: 1$ ) to afford 2dA and 2dB (10 mg, $24 \%$, colorless oil) as a 94:6 diastereomeric mixture. 
A: ${ }^{1} \mathbf{H}$ NMR $\left(300 \mathrm{MHz}, \mathrm{CDCl}_{3}\right): 7.41(\mathrm{~m}, 1 \mathrm{H}, \mathrm{H}-3), 7.08(\mathrm{~s}, 2 \mathrm{H}, \mathrm{Ar}), 4.18$ (dd, J=1.6, 6.5 Hz, 1H, H-5), 3.73 (m, 2H, $\left.\underline{\mathrm{H}}\left(\mathrm{CH}_{3}\right)_{2}\right), 3.39$ (m, 1H, H-4), 2.95 (m, 1H, H-4), 2.90 (m, 1H, $\left.\mathrm{CH}\left(\mathrm{CH}_{3}\right)_{2}\right), 2.16$ (m, 2H, H-1'), 1.51-

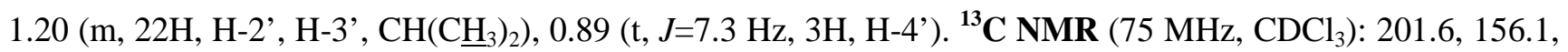
152.7, 150.1, 146.4, 134.0, 123.0, 66.3, 34.3, 29.6, 28.3, 28.1, 24.6, 23.7, 23.6, 22.3, 13.7. HRMS (EI+) calcd for $\left(\mathrm{C}_{24} \mathrm{H}_{36} \mathrm{O}_{2} \mathrm{~S}\right)[\mathrm{M}]^{+}$388.2436, found 388.2438.

B: ${ }^{1} \mathbf{H}$ NMR (300 MHz, $\mathrm{CDCl}_{3}$ ) (significant signal): 4.12 (m, 1H, H-5).

\section{2-n-Butyl- 5-(tert-butylsulfinyl)-2-cyclopentenone (2e):}

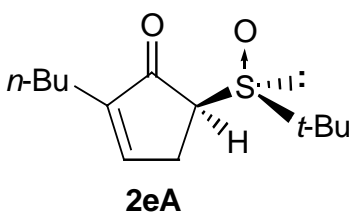

A solution of 1-hexyne $(39 \mu \mathrm{l}, 0.34 \mathrm{mmol})$ and $\mathrm{Co}_{2}(\mathrm{CO})_{8}(116 \mathrm{mg}, 0.34 \mathrm{mmol})$ in $\mathrm{CH}_{3} \mathrm{CN}(1 \mathrm{~mL})$ was stirred under argon atmosphere at rt. After $1 \mathrm{~h} N$ methylmorpholine $N$-oxide $(162 \mathrm{mg}, 1.38 \mathrm{mmol})$ and a solution of $1 \mathbf{e}(30 \mathrm{mg}, 0.23$ mmol) in $\mathrm{CH}_{3} \mathrm{CN}(1 \mathrm{~mL})$ were added. After $12 \mathrm{~h}$ at $\mathrm{rt}$ another 1.5 equivalents of the cobalt complex were added and the resulting solution was stirred for $12 \mathrm{~h}$. The reaction mixture was diluted with $\mathrm{CH}_{2} \mathrm{Cl}_{2}$, treated with trimethyl amine $\mathrm{N}$-oxide $(150 \mathrm{mg}$ ) and stirred for $10 \mathrm{~min}$ at $\mathrm{rt}$. After removal of the solvent, the crude product was dissolved in diethyl ether and filtered trough a pad of Celite. The combined organic solvents were evaporated and the residue was purified by chromatography (hexane/ethyl acetate $3: 2$ ) to afford 2eA (10 mg, $20 \%$, colorless oil).

${ }^{1}$ H NMR (300 MHz, $\mathrm{CDCl}_{3}$ ): 7.38 (m, 1H, H-3), 4.29 (dd, J=2.0, 6.9 Hz, 1H, H-5), 3.25 (m, 1H, H-4), 2.54 (m, 1H, H-4), 2.18 (m, 2H, H-1'), 1.45 (m, 2H, H-2'), 1.31 (m, 2H, H-3'), 1.27 (s, 9H, C(CH$\left.)_{3}\right) 0.89$ (t, J=7.3 Hz, 3H, H-4'). ${ }^{13} \mathbf{C}$ NMR (75 MHz, $\mathrm{CDCl}_{3}$ ): 204.7, 158.2, 145.9, 58.1, 55.1, 29.5, 24.6, 23.7, 23.4, 22.3, 13.8 . HRMS (EI+) calcd for $\left(\mathrm{C}_{9} \mathrm{H}_{14} \mathrm{O}_{2} \mathrm{~S}\right)[\mathrm{M}-t \mathrm{Bu}]^{+} 186.0715$, found 186.0717 .

\section{2-n-Butyl- 5-[2-(N,N-dimethylamino)phenylsulfinyl]-2-cyclopentenone (2f):}

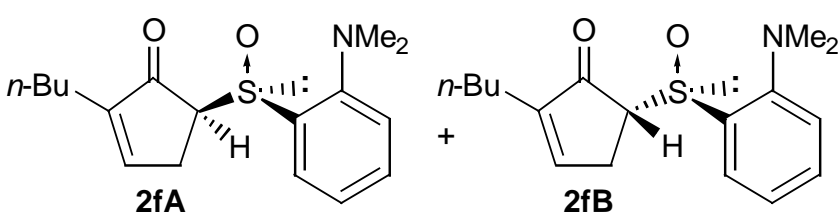

A solution of 1-hexyne $(23 \mu \mathrm{l}, 0.20 \mathrm{mmol})$ and $\mathrm{Co}_{2}(\mathrm{CO})_{8}(68 \mathrm{mg}, 0.20 \mathrm{mmol})$ in $\mathrm{CH}_{3} \mathrm{CN}(1 \mathrm{~mL})$ was stirred under argon atmosphere at rt. After $1 \mathrm{~h} \mathrm{~N}$ methylmorpholine $N$-oxide (108 $\mathrm{mg}, 0.92 \mathrm{mmol})$ and

a solution of $\mathbf{1 f}(30 \mathrm{mg}, 0.15 \mathrm{mmol})$ in $\mathrm{CH}_{3} \mathrm{CN}(1 \mathrm{~mL})$ were added at $0{ }^{\circ} \mathrm{C}$ and the resulting solution was stirred for $4 \mathrm{~h}$ at this temperature. The reaction mixture was diluted with $\mathrm{CH}_{2} \mathrm{Cl}_{2}$, treated with trimethyl amine $N$-oxide $(50 \mathrm{mg}$ ) and stirred for $10 \mathrm{~min}$ at $\mathrm{rt}$. After removal of the solvent, the crude product was dissolved in diethyl ether and filtered trough a pad of Celite. The combined organic solvents were evaporated and the residue was purified by chromatography $\left(\mathrm{Et}_{3} \mathrm{~N}\right.$ pretreated $\mathrm{SiO}_{2}$; hexane/ethyl acetate 3:1) to afford $\mathbf{2} \mathbf{f A}$ and $\mathbf{2 f B}$ (34 mg, 74 $\%$, colorless oil) as a 93:7 diastereomeric mixture.

A: ${ }^{1} \mathbf{H}$ NMR $\left(200 \mathrm{MHz}, \mathrm{CDCl}_{3}\right): 7.79(\mathrm{dd}, J=1.6,7.7 \mathrm{~Hz}, 1 \mathrm{H}, \mathrm{Ar}), 7.42$ (dt, J=1.6, 7.7 Hz, 1H, Ar), 7.30 (m, 1H, H-3), 7.24 (dt, J=1.2, 7.3 Hz, 1H, Ar), 7.09 (dd, J=1.2, 8.1 Hz, 1H, Ar), 4.29 (dd, J=2.4, 6.9 Hz, 1H, H-5), 2.77 (s, 6H, N( $\left.\left(\mathrm{CH}_{3}\right)_{2}\right), 2.63$ (m, 1H, H-4), 2.18 (m, 2H, H-1'), 2.03 (m, 1H, H-4), 1.50-1.24 (m, 4H, H-2', H- 
3'), 0.88 (t, $J=7.3 \mathrm{~Hz}, 3 \mathrm{H}, \mathrm{H}-4$ '). ${ }^{13} \mathbf{C}$ NMR (75 MHz, $\left.\mathrm{CDCl}_{3}\right)$ : 203.2, 158.0, 150.3, 146.9, 135.4, 131.6, 125.6, 123.7, 119.3, 63.0, 44.6, 29.5, 24.7, 23.6, 22.3, 13.8. HRMS $(\mathrm{FAB}+)$ calcd for $\left(\mathrm{C}_{17} \mathrm{H}_{24} \mathrm{NO}_{2} \mathrm{~S}\right)[\mathrm{M}+\mathrm{H}]^{+} 306.1528$, found 306.1532 .

B: ${ }^{1} \mathbf{H}$ NMR (200 MHz, $\left.\mathrm{CDCl}_{3}\right)$ (significant signals): 4.05 (m, 1H, H-5), 2.68 (s, 6H, N( $\left.\left(\mathrm{CH}_{3}\right)_{2}\right)$.

\section{2-tert-Butyl-5-[2-(N,N-dimethylamino)phenylsulfinyl]-2-cyclopentenone (3):}

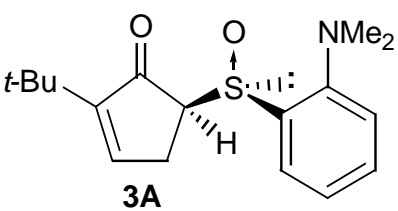

$3 A$ A solution of 3,3-dimethylbutyne $(28 \mu \mathrm{l}, 0.23 \mathrm{mmol})$ and $\mathrm{Co}_{2}(\mathrm{CO})_{8}(79 \mathrm{mg}, 0.23$ mmol) in $\mathrm{CH}_{3} \mathrm{CN}(1 \mathrm{~mL})$ was stirred under argon atmosphere at rt. After $1 \mathrm{~h} \mathrm{~N}$ methylmorpholine $N$-oxide (108 mg, $0.92 \mathrm{mmol}$ ) and a solution of $\mathbf{1 f}$ (30 mg, 0.15 $\mathrm{mmol})$ in $\mathrm{CH}_{3} \mathrm{CN}(1 \mathrm{~mL})$ were added at $\mathrm{rt}$. After $12 \mathrm{~h}$ another 1.5 equivalents of the cobalt complex were added and the resulting solution was stirred for $14 \mathrm{~h}$. The reaction mixture was diluted with $\mathrm{CH}_{2} \mathrm{Cl}_{2}$, treated with trimethyl amine $\mathrm{N}$-oxide $(100 \mathrm{mg})$ and stirred for $10 \mathrm{~min}$ at $\mathrm{rt}$. After removal of the solvent, the crude product was dissolved in diethyl ether and filtered trough a pad of Celite. The combined organic solvents were evaporated and the residue was purified by chromatography $\left(\mathrm{Et}_{3} \mathrm{~N}\right.$ pretreated $\mathrm{SiO}_{2}$; hexane/ethyl acetate 4:1) to afford 3A (26 mg, $55 \%$, white solid). M.p. 133-136 ${ }^{\circ} \mathrm{C} .{ }^{1} \mathbf{H}$ NMR (300 MHz, $\left.\mathrm{CDCl}_{3}\right): 7.80(\mathrm{dd}, J=1.6,7.7 \mathrm{~Hz}, 1 \mathrm{H}, \mathrm{Ar}), 7.43$ (dt, J=1.6, 7.3 Hz, 1H, Ar), 7.30 (t, J=2.8 Hz, 1H, H-3), 7.25 (dt, $J=1.2,7.7 \mathrm{~Hz}, 1 \mathrm{H}, \mathrm{Ar}), 7.10(\mathrm{dd}, J=1.2,8.1 \mathrm{~Hz}, 1 \mathrm{H}, \mathrm{Ar}), 4.27$ (dd, J=2.4, $6.9 \mathrm{~Hz}, 1 \mathrm{H}, \mathrm{H}-5), 2.78(\mathrm{~s}, 6 \mathrm{H}$, $\left.\mathrm{N}\left(\mathrm{CH}_{3}\right)_{2}\right), 2.60$ (td, J=2.8, $\left.19.0 \mathrm{~Hz}, 1 \mathrm{H}, \mathrm{H}-4\right), 1.99$ (ddd, J=2.8, 6.9, $\left.19.4 \mathrm{~Hz}, 1 \mathrm{H}, \mathrm{H}-4\right), 1.19\left(\mathrm{~s}, 9 \mathrm{H}, \mathrm{C}\left(\mathrm{CH}_{3}\right)_{3}\right)$. ${ }^{13}$ C NMR (75 MHz, $\left.\mathrm{CDCl}_{3}\right):$ 202.5, 156.6, 154.4, 150.4, 135.5, 131.6, 125.7, 123.7, 119.2, 64.1, 44.6, 32.1, 28.1, 22.8. HRMS (FAB+) calcd for $\left(\mathrm{C}_{17} \mathrm{H}_{24} \mathrm{NO}_{2} \mathrm{~S}\right)[\mathrm{M}+\mathrm{H}]^{+} 306.1528$, found 306.1536.

\section{2-Benzyl-5-[2-(N,N-dimethylamino)phenylsulfinyl]-2-cyclopentenone (4):}

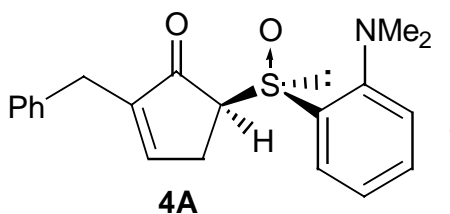

$4 \mathrm{~A}$

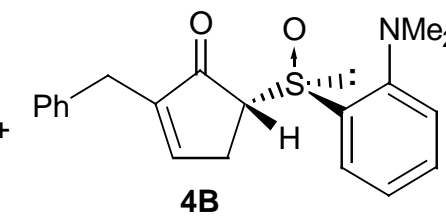

A solution of 3-phenyl-1-propyne (19 $\mu \mathrm{l}, 0.15$ $\mathrm{mmol})$ and $\mathrm{Co}_{2}(\mathrm{CO})_{8}(53 \mathrm{mg}, 0.15 \mathrm{mmol})$ in $\mathrm{CH}_{3} \mathrm{CN} \quad(1 \mathrm{~mL})$ was stirred under argon atmosphere at rt. After $1 \mathrm{~h} N$-methylmorpholine

After $5 \mathrm{~h}$ another 1.5 equivalents of the cobalt complex were added and the resulting solution was stirred for 9 h at $0{ }^{\circ} \mathrm{C}$. The reaction mixture was diluted with $\mathrm{CH}_{2} \mathrm{Cl}_{2}$, treated with trimethyl amine $N$-oxide $(70 \mathrm{mg})$ and stirred for $10 \mathrm{~min}$ at $\mathrm{rt}$. After removal of the solvent, the crude product was dissolved in diethyl ether and filtered trough a pad of Celite. The combined organic solvents were evaporated and the residue was purified by chromatography $\left(\mathrm{Et}_{3} \mathrm{~N}\right.$ pretreated $\mathrm{SiO}_{2}$; hexane/ethyl acetate 4:1) to afford $\mathbf{4 A}$ and $\mathbf{4 B}(20 \mathrm{mg}, 58 \%$, colorless oil), as a 93:7 diastereomeric mixture.

A: ${ }^{1}$ H NMR $\left(200 \mathrm{MHz}, \mathrm{CDCl}_{3}\right): 7.81(\mathrm{dd}, J=1.6,7.5 \mathrm{~Hz}, 1 \mathrm{H}, \mathrm{Ar}), 7.44(\mathrm{dt}, J=1.6,7.5 \mathrm{~Hz}, 1 \mathrm{H}, \mathrm{Ar}), 7.07-7.34$ (m, 8H, H-3, Ar), 4.36 (dd, J=2.7, 7.0 Hz, 1H, H-5), 3.53 (m, 2H, $\left.\mathrm{CH}_{2}\right), 2.78\left(\mathrm{~s}, 6 \mathrm{H}, \mathrm{N}\left(\mathrm{CH}_{3}\right)_{2}\right), 2.66(\mathrm{~m}, 1 \mathrm{H}, \mathrm{H}-$ 4), 2.01 (m, 1H, H-4). ${ }^{13} \mathrm{C}$ NMR (75 MHz, $\mathrm{CDCl}_{3}$ ): 202.4, 159.4, 150.4, 146.4, 138.1, 135.4, 131.7, 129.0, 
$128.5,126.4,125.7,123.8,119.3,63.2,44.6,31.5,23.6$. HRMS (FAB+) calcd for $\left(\mathrm{C}_{20} \mathrm{H}_{22} \mathrm{NO}_{2} \mathrm{~S}\right)[\mathrm{M}+\mathrm{H}]^{+}$ 340.1371 , found 340.1376 .

B: ${ }^{1} \mathbf{H}$ NMR (200 MHz, $\mathrm{CDCl}_{3}$ ) (significant signals): 4.11(m, 1H, H-5), $2.70\left(\mathrm{~s}, 6 \mathrm{H}, \mathrm{N}\left(\mathrm{CH}_{3}\right)_{2}\right)$.

\section{5-[2-(N,N-Dimethylamino)phenylsulfinyl]-2-p-tolyl-2-cyclopentenone (5):}

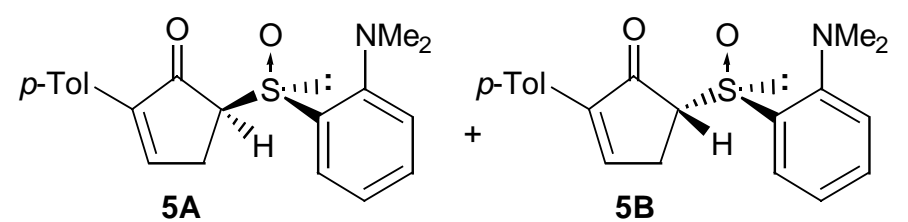

A solution of 4-ethynyltoluene $(29 \mu 1,0.23 \mathrm{mmol})$ and $\mathrm{Co}_{2}(\mathrm{CO})_{8}(79 \mathrm{mg}, 0.23 \mathrm{mmol})$ in $\mathrm{CH}_{3} \mathrm{CN}(1$ $\mathrm{mL}$ ) was stirred under argon atmosphere at rt. After $1 \mathrm{~h} N$-methylmorpholine $N$-oxide (108 mg, 0.92

mmol) and a solution of $1 \mathbf{f}(30 \mathrm{mg}, 0.15 \mathrm{mmol})$ in $\mathrm{CH}_{3} \mathrm{CN}(1 \mathrm{~mL})$ were added at $0{ }^{\circ} \mathrm{C}$ and the resulting solution was stirred for $12 \mathrm{~h}$ at this temperature. The reaction mixture was diluted with $\mathrm{CH}_{2} \mathrm{Cl}_{2}$, treated with trimethyl amine $\mathrm{N}$-oxide $(50 \mathrm{mg}$ ) and stirred for $10 \mathrm{~min}$ at $\mathrm{rt}$. After removal of the solvent, the crude product was dissolved in diethyl ether and filtered trough a pad of Celite. The combined organic solvents were evaporated and the residue was purified by chromatography $\left(\mathrm{Et}_{3} \mathrm{~N}\right.$ pretreated $\mathrm{SiO}_{2}$; hexane/ethyl acetate 3:1) to afford $\mathbf{5 A}$ and 5B (26 mg, $49 \%$, colorless oil) as a 93:7 diastereomeric mixture.

A: ${ }^{1} \mathbf{H}$ NMR $\left(300 \mathrm{MHz}, \mathrm{CDCl}_{3}\right): 7.85(\mathrm{dd}, J=1.6,7.7 \mathrm{~Hz}, 1 \mathrm{H}, \mathrm{Ar}), 7.80$ (t, $\left.J=2.8 \mathrm{~Hz}, 1 \mathrm{H}, \mathrm{H}-3\right), 7.62$ (d, $J=8.1$ $\mathrm{Hz}, 2 \mathrm{H}, p$-Tol), 7.46 (dt, J=1.6, $7.7 \mathrm{~Hz}, 1 \mathrm{H}, \mathrm{Ar}), 7.30$ (dd, J=1.2, $7.3 \mathrm{~Hz}, 1 \mathrm{H}, \mathrm{Ar}$ ), 7.18 (d, J=8.5 Hz, 2H, $p$ Tol), 7.13 (dd, J=1.2, 8.1 Hz, 1H, Ar), 4.48 (dd, J=2.8, 6.7 Hz, 1H, H-5), 2.83 (td, J=3.2, 19.8 Hz, 1H, H-4), $2.81\left(\mathrm{~s}, 6 \mathrm{H}, \mathrm{N}\left(\mathrm{CH}_{3}\right)_{2}\right), 2.35\left(\mathrm{~s}, 3 \mathrm{H}, \mathrm{ArC}_{3}\right), 2.19$ (ddd, J=2.8, 6.9, $\left.19.8 \mathrm{~Hz}, 1 \mathrm{H}, \mathrm{H}-4\right) .{ }^{13} \mathrm{C}$ NMR $(75 \mathrm{MHz}$, $\left.\mathrm{CDCl}_{3}\right): 201.3,158.3,150.4,143.5,138.6,135.4,131.7,129.1,128.1,126.9,125.7,123.8,119.3,64.4,44.6$, 23.2, 21.3. HRMS (EI+) calcd for $\left(\mathrm{C}_{20} \mathrm{H}_{21} \mathrm{NO}_{2} \mathrm{~S}\right)[\mathrm{M}]^{+} 339.1293$, found 339.1302.

B: ${ }^{1} \mathbf{H}$ NMR $\left(300 \mathrm{MHz}, \mathrm{CDCl}_{3}\right.$ ) (significant signals): 4.24 (dd, J=2.4, 6.1 Hz, 1H, H-5), $2.59\left(\mathrm{~s}, 6 \mathrm{H}, \mathrm{N}\left(\mathrm{CH}_{3}\right)_{2}\right)$, $2.32\left(\mathrm{~s}, 3 \mathrm{H}, \mathrm{ArC}_{3}\right)$.

\section{5-[2-(N,N-Dimethylamino)phenylsulfinyl]-2-trimethylsilyl-2-cyclopentenone (6):}

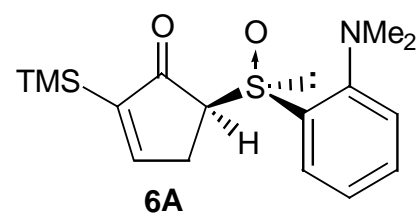

$6 \mathrm{~A}$

A solution of trimethylsilylacetylene $(43 \mu \mathrm{l}, 0.31 \mathrm{mmol})$ and $\mathrm{Co}_{2}(\mathrm{CO})_{8}(105 \mathrm{mg}$, $0.31 \mathrm{mmol})$ in $\mathrm{CH}_{3} \mathrm{CN}(1 \mathrm{~mL})$ was stirred under argon atmosphere at rt. After $1 \mathrm{~h}$ $\mathrm{N}$-methylmorpholine $\mathrm{N}$-oxide (72 $\mathrm{mg}, 0.62 \mathrm{mmol})$ and a solution of $\mathbf{1 f}(30 \mathrm{mg}$, $0.15 \mathrm{mmol})$ in $\mathrm{CH}_{3} \mathrm{CN}(1 \mathrm{~mL})$ were added at $\mathrm{rt}$ and the resulting solution was

stirred for $16 \mathrm{~h}$ at this temperature. The reaction mixture was diluted with $\mathrm{CH}_{2} \mathrm{Cl}_{2}$, treated with trimethyl amine $\mathrm{N}$-oxide $(70 \mathrm{mg}$ ) and stirred for $10 \mathrm{~min}$ at $\mathrm{rt}$. After removal of the solvent, the crude product was dissolved in diethyl ether and filtered trough a pad of Celite. The combined organic solvents were evaporated and the residue was purified by chromatography $\left(\mathrm{Et}_{3} \mathrm{~N}\right.$ pretreated $\mathrm{SiO}_{2}$; hexane/ethyl acetate 4:1) to afford $\mathbf{6 A}(29 \mathrm{mg}$, $59 \%$, white solid). M.p. 133-135 ${ }^{\circ} \mathrm{C} .{ }^{1} \mathbf{H}$ NMR (300 MHz, $\left.\mathrm{CDCl}_{3}\right): 7.82-7.79$ (m, 2H, Ar, H-3), 7.44 (dt, J=1.6, $7.3 \mathrm{~Hz}, 1 \mathrm{H}, \mathrm{Ar}), 7.26$ (t, J=1.2, $7.7 \mathrm{~Hz}, 1 \mathrm{H}, \mathrm{Ar}), 7.10$ (dt, J=1.2, 7.7 Hz, 1H, Ar), 4.28 (dd, J=2.8, 6.9 Hz, 1H, H-5), 2.81 (dt, J=3.2, $19.4 \mathrm{~Hz}, 1 \mathrm{H}, \mathrm{H}-4), 2.79$ (s, 6H, N( $\left.\left.\mathrm{CH}_{3}\right)_{2}\right), 2.16$ (ddd, J=2.4, 6.9, 19.8 Hz, 1H, H-4), 0.19 
(s, 9H, $\left.\mathrm{Si}\left(\mathrm{CH}_{3}\right)_{3}\right) .{ }^{13} \mathrm{C}$ NMR $\left(75 \mathrm{MHz}, \mathrm{CDCl}_{3}\right): 206.8,172.6,150.3,147.7,135.7,131.6,125.5,123.7,119.2$, 63.6, 44.5, 27.2, -2.0. HRMS (EI+) calcd for $\left(\mathrm{C}_{16} \mathrm{H}_{23} \mathrm{NO}_{2} \mathrm{SiS}\right)[\mathrm{M}]^{+} 321.1219$, found 321.1221.

\section{5-[2-(N,N-Dimethylamino)phenylsulfinyl]-2-[2-(triisopropylsilyloxy)ethyl)-2-cyclopentenone (7):}

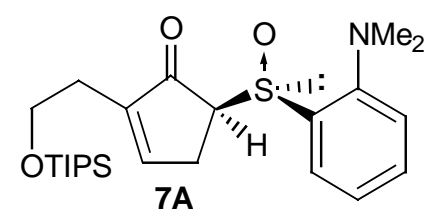

A solution of 4-(triisopropylsilyloxy)-1-butyne (35 mg, $0.15 \mathrm{mmol})$ and $\mathrm{Co}_{2}(\mathrm{CO})_{8}$ (53 mg, $0.15 \mathrm{mmol})$ in $\mathrm{CH}_{3} \mathrm{CN}(1 \mathrm{~mL})$ was stirred under argon atmosphere at $\mathrm{rt}$. After $1 \mathrm{~h} N$-methylmorpholine $N$-oxide $(72 \mathrm{mg}, 0.61 \mathrm{mmol})$ and a solution of $\mathbf{1 f}$ (20 mg, $0.10 \mathrm{mmol})$ in $\mathrm{CH}_{3} \mathrm{CN}(1 \mathrm{~mL})$ were added at $0{ }^{\circ} \mathrm{C}$ and the resulting solution was stirred for $7 \mathrm{~h}$ at this temperature. The reaction mixture was diluted with $\mathrm{CH}_{2} \mathrm{Cl}_{2}$, treated with trimethyl amine $\mathrm{N}$-oxide (40 mg) and stirred for $10 \mathrm{~min}$ at $\mathrm{rt}$. After removal of the solvent, the crude product was dissolved in diethyl ether and filtered trough a pad of Celite. The combined organic solvents were evaporated and the residue was purified by chromatography $\left(\mathrm{Et}_{3} \mathrm{~N}\right.$ pretreated $\mathrm{SiO}_{2}$; hexane/ethyl acetate 5:1) to afford 7A (30 mg, $66 \%$, white solid). M.p. 82-83 ${ }^{\circ} \mathrm{C} .{ }^{1} \mathbf{H}$ NMR (200 MHz, $\mathrm{CDCl}_{3}$ ): 7.81 (dd, J=1.6, $7.7 \mathrm{~Hz}$, 1H, Ar), 7.49 (m, 1H, H-3), 7.44 (dt, $J=1.6,7.7 \mathrm{~Hz}, 1 \mathrm{H}, \mathrm{Ar}), 7.26$ (dt, J=1.2, 7.7 Hz, 1H, Ar), 7.11 (dd, $J=1.2$, $7.7 \mathrm{~Hz}, 1 \mathrm{H}, \mathrm{Ar}), 4.30$ (dd, J=2.8, $6.9 \mathrm{~Hz}, 1 \mathrm{H}, \mathrm{H}-5), 3.80$ (m, 2H, H-2'), 2.78 (s, 6H, N( $\left.\left.\mathrm{CH}_{3}\right)_{2}\right), 2.70$ (m, 1H, H4), 2.47 (m, 2H, H-1'), 2.05 (m, 1H, H-4), 1.03 (m, 21H, TIPS). ${ }^{13} \mathbf{C}$ NMR (75 MHz, CDCl ${ }_{3}$ ): 203.0, 160.1, 150.4, 143.8, 135.6, 131.6, 125.7, 123.8, 119.3, 63.0, 61.2, 44.6, 28.7, 23.8, 18.0, 11.9. HRMS (FAB+) calcd for $\left(\mathrm{C}_{24} \mathrm{H}_{40} \mathrm{NO}_{3} \mathrm{SiS}\right)[\mathrm{M}+\mathrm{H}]^{+} 450.2498$, found 450.2497 .

$(\mathbf{5 S}, \mathbf{S R})-\mathbf{7 A}:[\boldsymbol{\alpha}]_{\mathbf{D}}=+290\left(c\right.$ 0.3, $\left.\mathrm{CHCl}_{3}\right)$. HPLC: ee > 99\% [Daicel Chiralcel OD column, hexane/isopropanol $98: 2,0.5 \mathrm{mLmin}^{-1}, \lambda=230 \mathrm{~nm} ; t_{\mathrm{R}}=39.8 \min (5 S, \mathrm{~S} R)$ and $\left.53.4 \mathrm{~min}(5 R, \mathrm{~S} S)\right]$.

\section{2-(3-Bromopropyl)-5-[2-(N,N-dimethylamino)phenylsulfinyl]-2-cyclopentenone (8):}

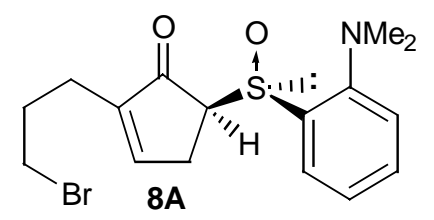

A solution of 1-bromo-4-pentyne $(79 \mathrm{mg}, 0.54 \mathrm{mmol})$ and $\mathrm{Co}_{2}(\mathrm{CO})_{8}(184 \mathrm{mg}$, $0.54 \mathrm{mmol})$ in $\mathrm{CH}_{3} \mathrm{CN}(3 \mathrm{~mL})$ was stirred under argon atmosphere at rt. After $1 \mathrm{~h}$ $\mathrm{N}$-methylmorpholine $\mathrm{N}$-oxide $(252 \mathrm{mg}, 2.15 \mathrm{mmol})$ and a solution of $\mathbf{1 f}(70 \mathrm{mg}$, $0.36 \mathrm{mmol})$ in $\mathrm{CH}_{3} \mathrm{CN}(2 \mathrm{~mL})$ were added at $0{ }^{\circ} \mathrm{C}$ and the resulting solution was stirred for $6 \mathrm{~h}$ at this temperature. The reaction mixture was diluted with $\mathrm{CH}_{2} \mathrm{Cl}_{2}$, treated with trimethyl amine $\mathrm{N}$-oxide $(120 \mathrm{mg}$ ) and stirred for $10 \mathrm{~min}$ at $\mathrm{rt}$. After removal of the solvent, the crude product was dissolved in diethyl ether and filtered trough a pad of Celite. The combined organic solvents were evaporated and the residue was purified by chromatography $\left(\mathrm{Et}_{3} \mathrm{~N}\right.$ pretreated $\mathrm{SiO}_{2}$; hexane/ethyl acetate 3:1) to afford $\mathbf{8 A}(90 \mathrm{mg}$, $68 \%$, white solid). M.p. $97-99^{\circ} \mathrm{C} .{ }^{1} \mathbf{H}$ NMR $\left(300 \mathrm{MHz}, \mathrm{CDCl}_{3}\right.$ ): 7.81 (dd, J=1.6, $\left.7.7 \mathrm{~Hz}, 1 \mathrm{H}, \mathrm{Ar}\right), 7.45$ (dt, $J=1.6,7.6 \mathrm{~Hz}, 1 \mathrm{H}, \mathrm{Ar}), 7.41$ (m, 1H, H-3), 7.27 (dt, J=1.2, 7.7 Hz, 1H, Ar), 7.11 (dd, J=1.2, $7.7 \mathrm{~Hz}, 1 \mathrm{H}, \mathrm{Ar}$ ), 4.33 (dd, J=2.4, 6.9 Hz, 1H, H-5), 3.39 (t, J=6.9 Hz, 2H, H-3'), 2.79 (s, 6H, N( $\left.\left.\mathrm{CH}_{3}\right)_{2}\right), 2.67$ (m, 1H, H-4), 2.40 (m, 2H, H-1'), 2.13-2.00 (m, 3H, H-4, H-2'). $\left.{ }^{13} \mathbf{C} \mathbf{~ N M R ~ ( 5 0 ~ M H z , ~} \mathrm{CDCl}_{3}\right): 202.8,159.2,150.3,144.7,135.2$,

\footnotetext{
${ }^{8}$ Shi, L.; Narula, C. K.; Mak, K. T.; Kao, L.; Xu, Y.; Heck, R.F. J. Org. Chem. 1983, 48, 3894-3900.
} 
131.6, 125.5, 123.7, 119.3, 62.9, 44.5, 32.8, 30.0, 23.7, 23.6. HRMS (FAB+) calcd for $\left(\mathrm{C}_{16} \mathrm{H}_{21} \mathrm{NO}_{2} \mathrm{SBr}\right)[\mathrm{M}+\mathrm{H}]^{+}$ 370.0476 , found 370.0470 .

\section{2,3-Dimethyl-5-[2-(N,N-dimethylamino)phenylsulfinyl]-2-cyclopentenone (9):}

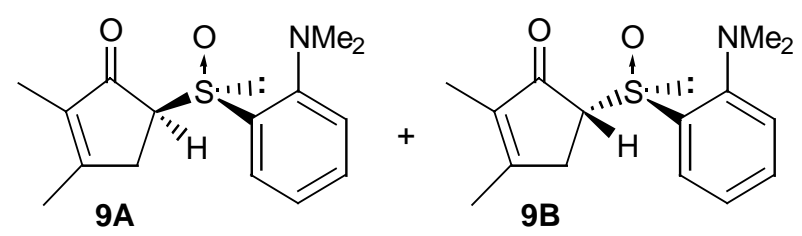

A solution of 2-butyne $(18 \mu \mathrm{L}, 0.23 \mathrm{mmol})$ and $\mathrm{Co}_{2}(\mathrm{CO})_{8}$ (79 $\mathrm{mg}, 0.23 \mathrm{mmol})$ in $\mathrm{CH}_{3} \mathrm{CN}(1 \mathrm{~mL})$ was stirred under argon atmosphere at $0{ }^{\circ} \mathrm{C}$. After $1 \mathrm{~h} N$-methylmorpholine $\mathrm{N}$-oxide (54 mg, $0.46 \mathrm{mmol})$ and a solution of $\mathbf{1 f}(15 \mathrm{mg}$,

$0.08 \mathrm{mmol})$ in $\mathrm{CH}_{3} \mathrm{CN}(0.5 \mathrm{~mL})$ were added at $0{ }^{\circ} \mathrm{C}$. The solution was placed in a Teflon reaction vessel and allowed to react at $10 \mathrm{Kbar}$ and $\mathrm{rt}$ for $48 \mathrm{~h}$. The reaction mixture was diluted with $\mathrm{CH}_{2} \mathrm{Cl}_{2}$, treated with trimethyl amine $N$-oxide $(50 \mathrm{mg}$ ) and stirred for $10 \mathrm{~min}$ at $\mathrm{rt}$. After removal of the solvent, the crude product was dissolved in diethyl ether and filtered trough a pad of Celite. The combined organic solvents were evaporated and the residue was purified by chromatography $\left(\mathrm{Et}_{3} \mathrm{~N}\right.$ pretreated $\mathrm{SiO}_{2}$; hexane/ethyl acetate 2:1) to afford 9A and 9B (7 mg, $33 \%)$ as a 92:8 diastereomeric mixture.

A: ${ }^{1} \mathbf{H}$ NMR $\left(200 \mathrm{MHz}, \mathrm{CDCl}_{3}\right): 7.81(\mathrm{dd}, J=1.6,7.7 \mathrm{~Hz}, 1 \mathrm{H}, \mathrm{Ar}), 7.43(\mathrm{dt}, J=1.6,7.3 \mathrm{~Hz}, 1 \mathrm{H}, \mathrm{Ar}), 7.26(\mathrm{t}$, $J=7.7 \mathrm{~Hz}, 1 \mathrm{H}, \mathrm{Ar}), 7.10$ (d, J=8.1 Hz, 1H, Ar), 4.28 (dd, J=2.8, 7.3 Hz, 1H, H-5), $2.78\left(\mathrm{~s}, 6 \mathrm{H}, \mathrm{N}\left(\mathrm{CH}_{3}\right)_{2}\right), 2.65-$ 2.56 (m, 1H, H-4), 2.01 (s, 3H, $\left.\mathrm{CH}_{3}\right), 1.97-1.81(\mathrm{~m}, 1 \mathrm{H}, \mathrm{H}-4), 1.72\left(\mathrm{~s}, 3 \mathrm{H}, \mathrm{CH}_{3}\right) .{ }^{13} \mathbf{C} \mathbf{~ N M R}\left(75 \mathrm{MHz}, \mathrm{CDCl}_{3}\right)$ : 202.6, 171.1, 150.4, 137.0, 135.7, 131.5, 125.7, 123.7, 119.2, 62.8, 44.6, 28.2, 17.3, 8.1. HRMS (EI+) calcd for $\left(\mathrm{C}_{15} \mathrm{H}_{19} \mathrm{NO}_{2} \mathrm{~S}\right)[\mathrm{M}]^{+}$277.1137, found 277.11530.

B: ${ }^{1} \mathbf{H}$ NMR $\left(200 \mathrm{MHz}, \mathrm{CDCl}_{3}\right)$ (significant signals): 4.06 (dd, J=3.2, $\left.4.9 \mathrm{~Hz}, 1 \mathrm{H}, \mathrm{H}-5\right), 2.68\left(\mathrm{~s}, 6 \mathrm{H}, \mathrm{N}\left(\mathrm{CH}_{3}\right)_{2}\right)$.

\section{SYNTHESIS OF (-)-PENTENOMYCIN I}

\section{$(1 R, 2 S)-2-(p$-Tolylsulfonylamino)-1-phenylpropyl $(S)-2-(N, N$-dimethylamino)phenyl sulfinate:}

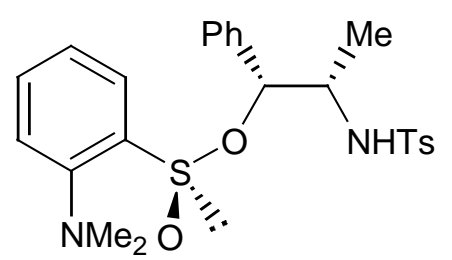

A solution of $N, N$-dimethyl-2-iodoaniline $(1.02 \mathrm{~g}, 4.13 \mathrm{mmol})$ and $\mathrm{I}_{2}$ in diethyl ether (4 mL) was added to $\mathrm{Mg}(150 \mathrm{mg}, 6.19 \mathrm{mmol})$ at $\mathrm{rt}$ under Ar atmosphere. The reaction mixture was heated until complete formation of the magnesium iodide. Then, it was diluted with THF $(15 \mathrm{~mL})$, cooled to $-78^{\circ} \mathrm{C}$ and a solution of 4-phenyl-5-methyl-3- $N$-tosylnorephedrine-1,2,3-oxathiazolidine-2-oxide $\left.{ }^{9} 7725 \mathrm{mg}, 2.06 \mathrm{mmol}\right)$ in $\mathrm{THF}(10$ $\mathrm{mL})$ was added. The solution was stirred at $-78{ }^{\circ} \mathrm{C}$ for $1 \mathrm{~h}$ and saturated aqueous $\mathrm{NH}_{4} \mathrm{Cl}(20 \mathrm{~mL})$ was then added. The mixture was extracted with ethyl acetate $(2 \times 15 \mathrm{~mL})$ and the combined organic layers were dried $\left(\mathrm{Na}_{2} \mathrm{SO}_{4}\right)$ and evaporated. The crude product was purified by flash chromatography (hexane/ethyl acetate $\left.4: 1\right)$ to afford the sulfinate (975 mg, $99 \%$, colorless oil). ${ }^{1} \mathbf{H} \mathbf{~ N M R ~ ( 2 0 0 ~ M H z , ~} \mathrm{CDCl}_{3}$ ): 7.99 (dd, J=1.6, 8.1 Hz, 1H, Ar), $7.83(\mathrm{~d}, J=8.1 \mathrm{~Hz}, 2 \mathrm{H}, \mathrm{Ar}), 7.51$ (dt, J=1.6, 7.5 Hz, 1H, Ar), 7.35-7.20 (m, 6H, Ar), 7.09 (m, 3H, Ar), 5.70

\footnotetext{
${ }^{9}$ Han, Z.; Krishnamurthy, D.; Grover, P.; Wilkinson, H. S.; Fang, Q. K.; Su, X.; Lu, Z.-H.; Magiera, D.; Senanayake, C. H. Angew. Chem. Int. Ed. 2003, 42, 2032-2035.
} 
(d, J=9.7 Hz, 1H, H-1), 5.11 (d, J=2.7 Hz, 1H, NH), 3.64 (m, 1H, H-2), 2.62 (s, 6H, N( $\left.\left.\mathrm{CH}_{3}\right)_{2}\right), 2.43$ (s, 3H, $\left.\mathrm{ArCH}_{3}\right), 0.93$ (d, J=7.0 Hz, 3H, H-3). ${ }^{13} \mathbf{C}$ NMR (75 MHz, $\left.\mathrm{CDCl}_{3}\right): 152.4,143.2,138.6,138.3,137.5,133.5$, 129.6, 128.2, 127.9, 127.1, 125.9, 125.0, 123.2, 119.7, 84.1, 54.3, 45.1, 21.5, 14.7. HRMS (FAB+) calcd for $\left(\mathrm{C}_{24} \mathrm{H}_{29} \mathrm{~N}_{2} \mathrm{O}_{4} \mathrm{~S}_{2}\right)[\mathrm{M}+\mathrm{H}]^{+} 473.1569$, found 473.1573. [ $\left.\alpha\right]_{\mathrm{D}}=-147\left(\right.$ c 1.2, $\left.\mathrm{CHCl}_{3}\right)$.

\section{$(R)-2-(N, N$-Dimethylamino)phenyl vinyl sulfoxide $[(R)-1 f]$ :}

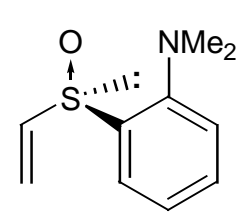

Vinylmagnesium bromide (1 M in THF, $5.24 \mathrm{~mL}, 5.24 \mathrm{mmol}$ ) was added to a solution of sulfinate $(825 \mathrm{mg}, 1.75 \mathrm{mmol})$ in THF $(15 \mathrm{~mL})$ at $-78^{\circ} \mathrm{C}$. The solution was stirred at $-78^{\circ} \mathrm{C}$ for $2 \mathrm{~h}$ and saturated aqueous $\mathrm{NH}_{4} \mathrm{Cl}(20 \mathrm{~mL})$ was then added. The mixture was extracted with ethyl acetate $(2 \times 15 \mathrm{~mL})$ and the combined organic layers were dried $\left(\mathrm{Na}_{2} \mathrm{SO}_{4}\right)$ and evaporated. The crude product was purified by flash chromatography (hexane/diethyl ether 1:1) to afford the sulfoxide $(\boldsymbol{R})-\mathbf{1 f}(225 \mathrm{mg}, 66 \%$, colorless oil). The spectral data were identical to those described for $( \pm)-\mathbf{1 f}$. $[\alpha]_{\mathbf{D}}=+551\left(c 1, \mathrm{CHCl}_{3}\right)$. HPLC: ee > 99\% [Daicel Chiralpak AD column, hexane/isopropanol 95:5, 0.5 $\operatorname{mLmin}^{-1}, \lambda=254 \mathrm{~nm} ; t_{\mathrm{R}}=19.5 \min (S)$ and $\left.21.4 \min (R)\right]$.

(5S,SR)-5-[2-(N,N-Dimethylamino)phenylsulfinyl]-2-(triisopropylsilyloxy)methyl-2-cyclopentenone (12):

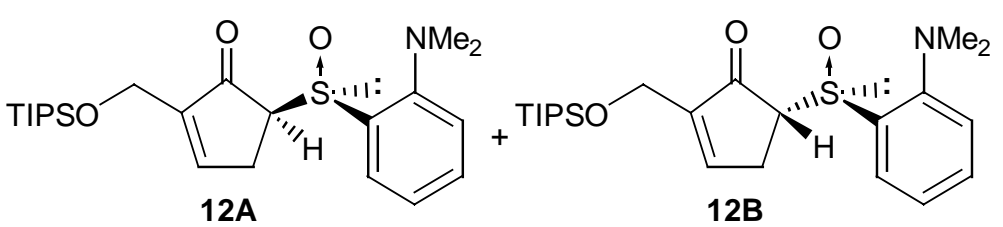
A solution of 3-(triisopropylsilyloxy)-1propyne (244 $\mathrm{mg}, 1.15 \mathrm{mmol})$ and $\mathrm{Co}_{2}(\mathrm{CO})_{8}$ (394 mg, $1.15 \mathrm{mmol})$ in $\mathrm{CH}_{3} \mathrm{CN}(3 \mathrm{~mL})$ was stirred under argon atmosphere at rt. After 1 h $\mathrm{N}$-methylmorpholine $\mathrm{N}$-oxide $(540 \mathrm{mg}, 4.61 \mathrm{mmol})$ and a solution of $(\boldsymbol{R})$-1f $(150 \mathrm{mg}, 0.77 \mathrm{mmol})$ in $\mathrm{CH}_{3} \mathrm{CN}$ $(2 \mathrm{~mL})$ were added at $0{ }^{\circ} \mathrm{C}$ and the resulting solution was stirred for $2 \mathrm{~h}$ at this temperature. The reaction mixture was diluted with $\mathrm{CH}_{2} \mathrm{Cl}_{2}$, treated with trimethyl amine $\mathrm{N}$-oxide $(250 \mathrm{mg}$ ) and stirred for $10 \mathrm{~min}$ at $\mathrm{rt}$. After removal of the solvent, the crude product was dissolved in diethyl ether and filtered trough a pad of Celite. The combined organic solvents were evaporated and the residue was purified by chromatography $\left(\mathrm{Et}_{3} \mathrm{~N}\right.$ pretreated $\mathrm{SiO}_{2}$; hexane/ethyl acetate 6:1) to afford 12A and 12B (207 $\mathrm{mg}, 62 \%$, white solid) as a 93:7 diastereomeric mixture. The mixture was triturated with cold hexane to afford diastereomerically pure $\mathbf{1 2 A}$ (176 mg, $53 \%$ overall yield).

A : M.p. $87-88^{\circ} \mathrm{C} .{ }^{1} \mathbf{H}$ NMR $\left(300 \mathrm{MHz}, \mathrm{CDCl}_{3}\right): 7.83(\mathrm{dd}, J=1.6,7.7 \mathrm{~Hz}, 1 \mathrm{H}, \mathrm{Ar}), 7.60$ (m, 1H, H-3), 7.45 (dt, $J=1.6,7.7 \mathrm{~Hz}, 1 \mathrm{H}, \mathrm{Ar}), 7.27$ (dt, J=1.2, $7.7 \mathrm{~Hz}, 1 \mathrm{H}, \mathrm{Ar}), 7.11$ (dd, J=1.2, 8.1 Hz, 1H, Ar), 4.47 (m, 2H, H-1'), 4.36 (dd, J=2.4, 6.9 Hz, 1H, H-5), 2.78 (s, 6H, N( $\left.\left.\mathrm{CH}_{3}\right)_{2}\right), 2.78-2.67$ (m, 1H, H-4), 2.17-2.04 (m, 1H, H-4), 1.05 (m, 21H, TIPS). ${ }^{13} \mathbf{C}$ NMR (75 MHz, $\mathrm{CDCl}_{3}$ ): 201.6, 158.5, 150.4, 147.0, 135.4, 131.7, 125.7, 123.8, 119.3, 64.0, 58.5, 44.6, 23.9, 17.9, 11.8. HRMS (FAB+) calcd for $\left(\mathrm{C}_{23} \mathrm{H}_{38} \mathrm{NO}_{3} \mathrm{SiS}\right)[\mathrm{M}+\mathrm{H}]^{+} 436.2342$, found 436.2338. $[\alpha]_{\mathbf{D}}=+470\left(c \quad 0.2, \mathrm{CHCl}_{3}\right)$. HPLC: ee > 99\% [Daicel Chiralcel OD column, hexane/isopropanol 98:2, 0.5 $\operatorname{mLmin}^{-1}, \lambda=220 \mathrm{~nm} ; t_{\mathrm{R}}=24.2 \min (5 S, \mathrm{~S} R)$ and $\left.30.1 \min (5 R, \mathrm{~S} S)\right]$.

B: ${ }^{1} \mathbf{H}$ NMR (300 MHz, $\left.\mathrm{CDCl}_{3}\right)$ (significant signals): 4.11 (dd, J=4.0, $\left.4.9 \mathrm{~Hz}, 1 \mathrm{H}, \mathrm{H}-5\right), 2.68\left(\mathrm{~s}, 6 \mathrm{H}, \mathrm{N}\left(\mathrm{CH}_{3}\right)_{2}\right)$. 


\section{$(2 S, 3 S, 5 S, S R)$-[2-( $N, N$-Dimethylamino)phenylsulfinyl-2,3-osmadioxy-2-(triisopropylsilyloxy)methyl-2-}

cyclopentan-1-one]-[N,N,N',N'-tetramethylethylenediamine] osmate diester (13):

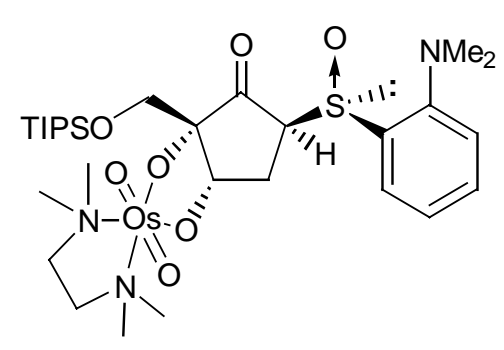

To a solution of cyclopentenone $\mathbf{1 2 A}(110 \mathrm{mg}, 0.25 \mathrm{mmol})$ and TMEDA ${ }^{10}(42$ $\mu \mathrm{L}, 0.28 \mathrm{mmol})$ in $\mathrm{CH}_{2} \mathrm{Cl}_{2}(3 \mathrm{~mL})$ cooled to $-78{ }^{\circ} \mathrm{C}$ under argon, was added a solution of $\mathrm{OsO}_{4}(71 \mathrm{mg}, 0.28 \mathrm{mmol})$ in $\mathrm{CH}_{2} \mathrm{Cl}_{2}(2 \mathrm{~mL})$. After stirring at $-78{ }^{\circ} \mathrm{C}$ for $1 \mathrm{~h}$, the reaction mixture was treated with sat. aq solution of $\mathrm{Na}_{2} \mathrm{SO}_{3}(5 \mathrm{~mL})$ and stirred for $2 \mathrm{~h}$ at $\mathrm{rt}$. The mixture was extracted with AcOEt $(5 \times 10 \mathrm{~mL})$ and the combined organic layers were dried $\left(\mathrm{Na}_{2} \mathrm{SO}_{4}\right)$ and evaporated. The crude product was triturated with hexane and filtered to afford $\mathbf{1 3}$ (165 mg, $81 \%$, brown solid). M.p. decomp > 140 ${ }^{\circ} \mathrm{C} .{ }^{1} \mathbf{H}$ NMR $\left(300 \mathrm{MHz}, \mathrm{CDCl}_{3}\right): 7.83(\mathrm{dd}, J=1.6,7.7 \mathrm{~Hz}, 1 \mathrm{H}, \mathrm{Ar}), 7.39$ (dt, J=1.6, $\left.7.7 \mathrm{~Hz}, 1 \mathrm{H}, \mathrm{Ar}\right), 7.25$ (dt, $J=1.2,7.3 \mathrm{~Hz}, 1 \mathrm{H}, \mathrm{Ar}$ ), 7.06 (dd, $J=1.2,7.7 \mathrm{~Hz}, 1 \mathrm{H}, \mathrm{Ar}$ ), 5.03 (dd, $J=1.6,4.4 \mathrm{~Hz}, 1 \mathrm{H}, \mathrm{H}-3$ ), 4.36 (dd, $J=8.9$, $11.3 \mathrm{~Hz}, 1 \mathrm{H}, \mathrm{H}-5), 4.23$ and 4.02 (AB system, $J=10.9 \mathrm{~Hz}, 1 \mathrm{H}, \mathrm{H}-1^{\prime}$ ), 3.02 (m, 4H, $\mathrm{NCH}_{2} \mathrm{CH}_{2} \mathrm{~N}$ ), 2.84 (s, 3H, $\left.\mathrm{N}\left(\mathrm{CH}_{3}\right)_{2}\right), 2.83\left(\mathrm{~s}, 3 \mathrm{H}, \mathrm{N}\left(\mathrm{CH}_{3}\right)_{2}\right), 2.82(\mathrm{~m}, 1 \mathrm{H}, \mathrm{H}-4), 2.80\left(\mathrm{~s}, 3 \mathrm{H}, \mathrm{N}\left(\mathrm{CH}_{3}\right)_{2}\right), 2.73\left(\mathrm{~s}, 3 \mathrm{H}, \mathrm{N}\left(\mathrm{CH}_{3}\right)_{2}\right), 2.68(\mathrm{~s}, 6 \mathrm{H}$, $\left.\mathrm{N}\left(\mathrm{CH}_{3}\right)_{2}\right), 1.76$ (m, 1H, H-4), 1.13 (m, 21H, TIPS). ${ }^{13} \mathbf{C}$ NMR $\left(75 \mathrm{MHz}, \mathrm{CDCl}_{3}\right): 212.5,150.3,136.5,131.0$, 125.7, 123.8, 119.3, 99.7, 88.7, 65.7, 64.3, 64.0, 61.8, 51.7, 51.4, 51.3, 46.0, 44.5, 23.8, 17.9, 11.8. HRMS $(\mathrm{FAB}+)$ calcd for $\left(\mathrm{C}_{29} \mathrm{H}_{54} \mathrm{~N}_{3} \mathrm{O}_{7} \mathrm{SiSOs}\right)[\mathrm{M}+\mathrm{H}]^{+} 808.3067$, found 808.3066. [ $\left.\alpha\right]_{\mathbf{D}}=-99\left(c 0.2, \mathrm{CHCl}_{3}\right)$.

\section{(2S,3S)-[2,3-Osmadioxy-2-(triisopropylsilyloxy)methyl-4-cyclopentenone]-[ $N, N, N^{\prime}, N^{\prime}-$}

\section{tetramethylethylenediamine] osmate diester (14):}

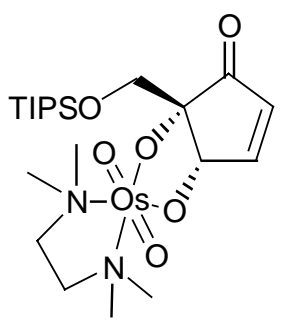

A solution of sulfoxide $13(110 \mathrm{mg}, 0.14 \mathrm{mmol})$ in toluene $(5 \mathrm{~mL})$ was heated to reflux for $1 \mathrm{~h}$. The mixture was then cooled to rt, solvent was evaporated at reduced pressure and the residue was purified by chromatography $\left(\mathrm{CH}_{3} \mathrm{CN}\right)$ to afford $14(64 \mathrm{mg}, 72 \%$, brown solid). M.p. decomp > $200{ }^{\circ} \mathrm{C} .{ }^{1} \mathbf{H}$ NMR $\left(300 \mathrm{MHz}, \mathrm{CDCl}_{3}\right): 7.61(\mathrm{dd}, J=2.0,5.7 \mathrm{~Hz}, 1 \mathrm{H}$, H-3), 6.26 (dd, $J=1.2,6.1 \mathrm{~Hz}, 1 \mathrm{H}, \mathrm{H}-2), 5.58$ (dd, $J=1.2,2.4 \mathrm{~Hz}, 1 \mathrm{H}, \mathrm{H}-4), 4.23$ and 4.00 (AB system, J=8.9 Hz, 1H, H-1'), 3.05 (m, 4H, $\left.\mathrm{NCH}_{2} \mathrm{CH}_{2} \mathrm{~N}\right), 2.86\left(\mathrm{~s}, 3 \mathrm{H}, \mathrm{N}\left(\mathrm{CH}_{3}\right)_{2}\right), 2.84$ (s, 3H, N( $\left.\left.\mathrm{CH}_{3}\right)_{2}\right)$, 2.83 (s, 3H, N( $\left.\left.\mathrm{CH}_{3}\right)_{2}\right), 2.75$ (s, 3H, N( $\left.\left.\mathrm{CH}_{3}\right)_{2}\right), 1.02$ (m, 21H, TIPS). ${ }^{13} \mathbf{C} \mathbf{N M R}\left(50 \mathrm{MHz}, \mathrm{CDCl}_{3}\right): 206.7,162.9$, 133.2, 94.1, 64.4, 62.3, 52.1, 51.6, 17.9, 11.9. HRMS (FAB+) calcd for $\left(\mathrm{C}_{21} \mathrm{H}_{43} \mathrm{~N}_{2} \mathrm{O}_{6} \mathrm{SiOs}\right)[\mathrm{M}+\mathrm{H}]^{+} 639.2505$, found 639.2487. $[\alpha]_{\mathbf{D}}=+245\left(c 0.1, \mathrm{CHCl}_{3}\right)$.

\section{(-)-Pentenomycin I:}

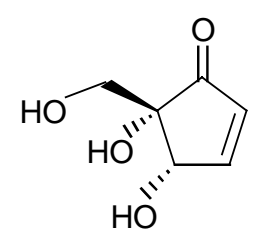

A solution of cyclopentenone $14(64 \mathrm{mg}, 0.10 \mathrm{mmol})$ in $\mathrm{HCl} 2 \mathrm{M}(4 \mathrm{~mL})$ was stirred at $\mathrm{rt}$ for 6 h. The mixture was washed with $\mathrm{CH}_{2} \mathrm{Cl}_{2}(3 \times 5 \mathrm{~mL})$ and the aqueous layer was concentrated. The residue was purified by chromatography (ethyl acetate/methanol 10:1) to afford (-)-(2S,

${ }^{10}$ Donohoe, T. J.; Blades, K.; Moore, P. R.; Waring, M. J.; Winter, J. J. G.; Helliwell, M.; Newcombe, N. J.; Stemp, G. J. Org. Chem. 2002, 67, 7946-7956. 
3S)-pentenomycin (11 mg, $76 \%$, colorless oil). ${ }^{1} \mathbf{H}$ NMR $\left(300 \mathrm{MHz}, \mathrm{D}_{2} \mathrm{O}\right): 7.78(\mathrm{dd}, J=2.4,6.1 \mathrm{~Hz}, 1 \mathrm{H}, \mathrm{H}-3)$, $6.39(\mathrm{dd}, J=1.2,6.1 \mathrm{~Hz}, 1 \mathrm{H}, \mathrm{H}-2), 4.78(\mathrm{dd}, J=1.2,2.8 \mathrm{~Hz}, 1 \mathrm{H}, \mathrm{H}-4), 3.77$ and 3.71 (AB system, $J=11.3 \mathrm{~Hz}, 2 \mathrm{H}$, H-6). ${ }^{13} \mathbf{C}$ NMR $\left(75 \mathrm{MHz}, \mathrm{D}_{2} \mathrm{O}\right): 213.3,168.0,136.9,79.8,75.1,66.8 .[\alpha]_{\mathbf{D}}=-32$ (c 0.2, EtOH) $\left[\right.$ natural $^{1 !} !$ $\left.[\alpha]_{\mathrm{D}}^{21}=-32(c 0.3, \mathrm{EtOH})\right]$.

\section{(-)-Pentenomycin I triacetate:}

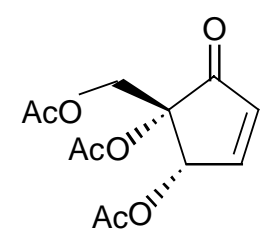

Acetic anhydride $(101 \mu \mathrm{L}, 1.07 \mathrm{mmol})$ was added to a solution of pentenomycin $(11 \mathrm{mg}$, $0.076 \mathrm{mmol})$ in ice-cold pyridine $(204 \mu \mathrm{L}, 2.52 \mathrm{mmol})$ under argon. After $24 \mathrm{~h}$ at $\mathrm{rt}$, water $(5$ $\mathrm{mL}$ ) was added and the mixture was extracted with ethyl acetate $(3 \times 5 \mathrm{~mL})$. The combined organic solvents were evaporated and the residue was purified by chromatography (hexane/ethyl acetate 2:1) to afford (-)-pentenomycin triacetate (13 mg, $63 \%$, white solid). M.p. $111-112^{\circ} \mathrm{C}$ [lit. ${ }^{1}$ : m.p. $112-114{ }^{\circ} \mathrm{C}$ ]. ${ }^{1} \mathbf{H}$ NMR $\left(300 \mathrm{MHz}, \mathrm{CDCl}_{3}\right.$ ): 7.45 (dd, $\left.J=2.8,6.1 \mathrm{~Hz}, 1 \mathrm{H}, \mathrm{H}-3\right), 6.51$ (dd, $J=1.6,6.5$ Hz, 1H, H-2), 5.81 (dd, J=1.6, 2.8 Hz, 1H, H-4), 4.36 (s, 2H, H-6), 2.10 (s, 3H, Ac), 2.08 (s, 3H, Ac), 2.05 (s, 3H, Ac). ${ }^{13}$ C NMR (75 MHz, $\left.\mathrm{CDCl}_{3}\right):$ 199.7, 170.0, 169.5, 168.6, 154.3, 135.8, 77.2, 72.1, 64.3, 20.6, 20.4, 20.1. $[\alpha]_{\mathbf{D}}=-8(c 0.2, \mathrm{EtOH})\left[\right.$ lit. $\left.^{12}:[\alpha]_{\mathrm{D}}=-8(c 0.5, \mathrm{EtOH})\right]$. HPLC: ee $>99 \%$ [Daicel Chiralpak AD column, hexane/isopropanol 95:5, $0.5 \mathrm{mLmin}^{-1}, \lambda=220 \mathrm{~nm} ; t_{\mathrm{R}}=33.0 \mathrm{~min}(2 S, 3 S)$ and $\left.36.5 \min (2 R, 3 R)\right]$.

\footnotetext{
${ }^{11}$ Umino, K.; Takeda, N.; Ito, Y.; Okuda, T. Chem. Pharm. Bull. 1974, 22, 1233-1238.

${ }^{12}$ Hetmanski, M.; Purcell, N.; Stoodley, R. J. J. Chem. Soc., Perkin Trans 1 1984, 2089-2096.
} 


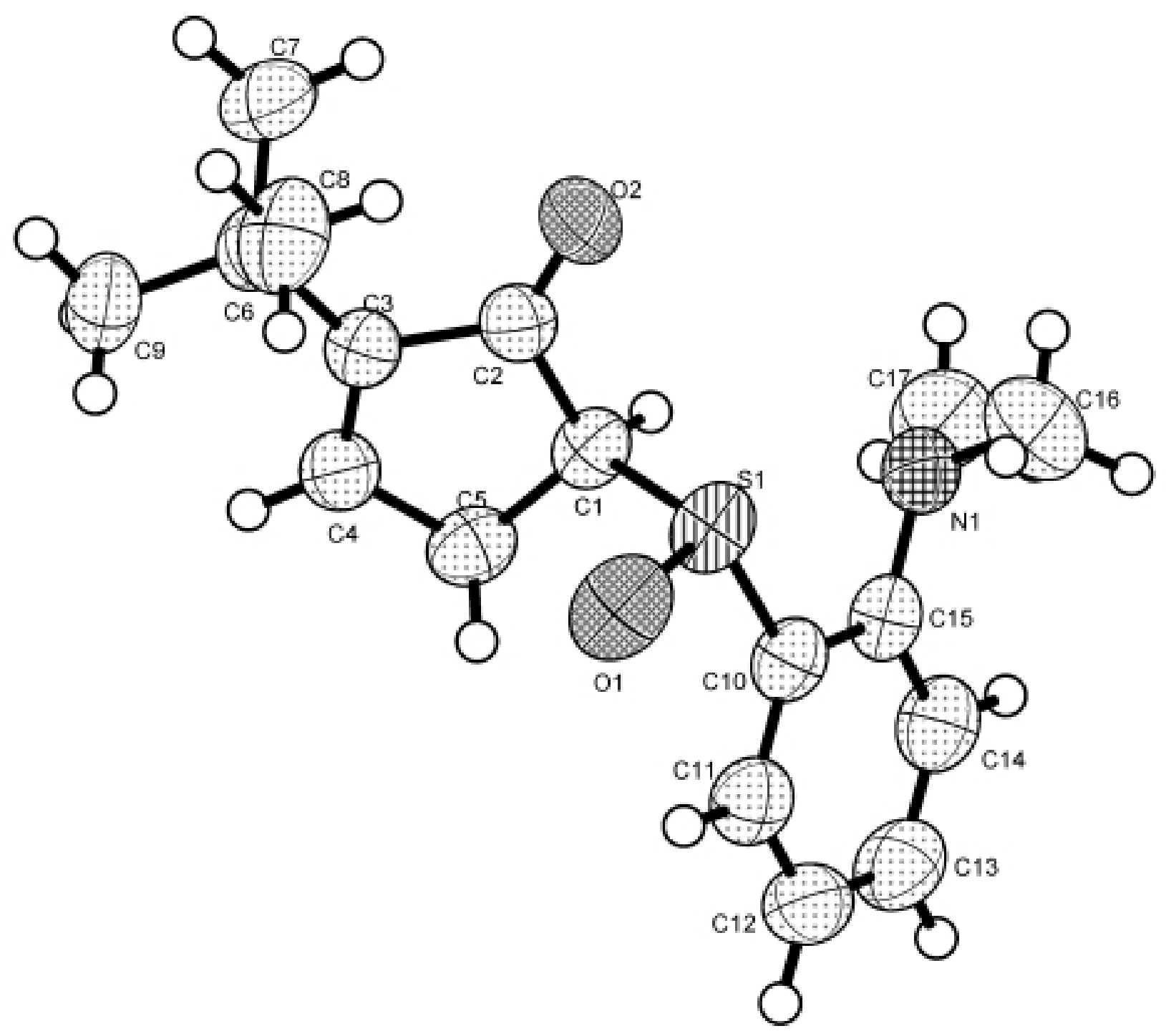


Table 1. Crystal data and structure refinement

Identification code

Empirical formula

Formula weight

Temperature

Wavelength

Crystal system

Space group

Unit cell dimensions

Volume

Z

Density (calculated)

Absorption coefficient

$\mathrm{F}(000)$

Crystal size

Theta range for data collection

Index ranges

Reflections collected

Independent reflections

Completeness to theta $=70.42^{\circ}$

Absorption correction

Refinement method

Data / restraints / parameters

Goodness-of-fit on $\mathrm{F}^{2}$

Final $\mathrm{R}$ indices [I>2sigma(I)]

$\mathrm{R}$ indices (all data)

Extinction coefficient

Largest diff. peak and hole mr158_m

C17 H23 N O2 S

305.42

296(2) K

$1.54178 \AA$

Orthorhombic

Pbca

$a=11.65520(10) \AA \quad \alpha=90^{\circ}$.

$\mathrm{b}=12.22290(10) \AA \quad \beta=90^{\circ}$.

$\mathrm{c}=24.1540(2) \AA \quad \gamma=90^{\circ}$.

3440.99(5) $\AA^{3}$

8

$1.179 \mathrm{Mg} / \mathrm{m}^{3}$

$1.696 \mathrm{~mm}^{-1}$

1312

$0.25 \times 0.20 \times 0.10 \mathrm{~mm}^{3}$

3.66 to $70.42^{\circ}$.

$-13<=\mathrm{h}<=13,-14<=\mathrm{k}<=14,-29<=\mathrm{l}<=27$

19283

$3225[\mathrm{R}(\mathrm{int})=0.0297]$

$98.1 \%$

YES, SADABS v. 2.03

Full-matrix least-squares on $\mathrm{F}^{2}$

3225 / 0 / 283

1.067

$\mathrm{R} 1=0.0340, \mathrm{wR} 2=0.1032$

$\mathrm{R} 1=0.0372, \mathrm{wR} 2=0.1074$

$0.00016(8)$

0.252 and -0.230 e. $\AA^{-3}$ 
Table 2. Atomic coordinates $\left(\times \mathbf{1 0}^{4}\right)$ and equivalent isotropic displacement parameters $\left(\AA^{2} \times 10^{3}\right)$. U(eq) is defined as one third of the trace of the orthogonalized $U^{i j}$ tensor.

\begin{tabular}{|c|c|c|c|c|}
\hline & $\mathrm{X}$ & $\mathrm{y}$ & $\mathrm{z}$ & $\mathrm{U}(\mathrm{eq})$ \\
\hline $\mathrm{N}(1)$ & $1776(1)$ & $2795(1)$ & $3437(1)$ & $66(1)$ \\
\hline $\mathrm{O}(1)$ & $-749(1)$ & $1185(1)$ & $2352(1)$ & $78(1)$ \\
\hline $\mathrm{O}(2)$ & $1462(1)$ & $2888(1)$ & $1588(1)$ & $78(1)$ \\
\hline$S(1)$ & $156(1)$ & $1886(1)$ & $2603(1)$ & $58(1)$ \\
\hline $\mathrm{C}(1)$ & 1511(1) & $1462(1)$ & $2286(1)$ & $51(1)$ \\
\hline$C(2)$ & $1525(1)$ & 1919(1) & 1699(1) & $53(1)$ \\
\hline $\mathrm{C}(3)$ & $1638(1)$ & $993(1)$ & 1313(1) & $50(1)$ \\
\hline $\mathrm{C}(4)$ & $1716(1)$ & $79(1)$ & $1609(1)$ & $55(1)$ \\
\hline$C(5)$ & 1639(1) & $235(1)$ & $2222(1)$ & $58(1)$ \\
\hline$C(6)$ & $1655(1)$ & 1133(1) & $688(1)$ & $59(1)$ \\
\hline$C(7)$ & $2659(2)$ & $1867(2)$ & $524(1)$ & $78(1)$ \\
\hline $\mathrm{C}(8)$ & $517(2)$ & $1648(2)$ & $505(1)$ & $85(1)$ \\
\hline $\mathrm{C}(9)$ & 1792(2) & $24(2)$ & $406(1)$ & $81(1)$ \\
\hline$C(10)$ & $427(1)$ & $1325(1)$ & $3280(1)$ & $51(1)$ \\
\hline $\mathrm{C}(11)$ & $-168(1)$ & $399(1)$ & $3439(1)$ & $61(1)$ \\
\hline$C(12)$ & $33(2)$ & $-52(1)$ & $3954(1)$ & $69(1)$ \\
\hline$C(13)$ & $829(2)$ & $419(1)$ & 4299(1) & $73(1)$ \\
\hline$C(14)$ & $1420(2)$ & $1346(1)$ & 4141(1) & $67(1)$ \\
\hline$C(15)$ & $1218(1)$ & 1835(1) & $3629(1)$ & $53(1)$ \\
\hline$C(16)$ & $1115(3)$ & $3785(2)$ & $3530(1)$ & $107(1)$ \\
\hline$C(17)$ & $2967(2)$ & $2908(3)$ & $3612(1)$ & $97(1)$ \\
\hline
\end{tabular}


Table 3. Bond lengths $[\AA ̊]$ and angles $\left[{ }^{\circ}\right]$.

\begin{tabular}{|c|c|}
\hline $\mathrm{N}(1)-\mathrm{C}(15)$ & $1.4196(18)$ \\
\hline $\mathrm{N}(1)-\mathrm{C}(16)$ & $1.452(3)$ \\
\hline $\mathrm{N}(1)-\mathrm{C}(17)$ & $1.457(3)$ \\
\hline $\mathrm{O}(1)-\mathrm{S}(1)$ & $1.4881(12)$ \\
\hline $\mathrm{O}(2)-\mathrm{C}(2)$ & $1.2165(16)$ \\
\hline$S(1)-C(10)$ & $1.8013(13)$ \\
\hline $\mathrm{S}(1)-\mathrm{C}(1)$ & $1.8295(13)$ \\
\hline $\mathrm{C}(1)-\mathrm{C}(5)$ & $1.515(2)$ \\
\hline $\mathrm{C}(1)-\mathrm{C}(2)$ & $1.5241(18)$ \\
\hline$C(2)-C(3)$ & $1.4725(18)$ \\
\hline$C(3)-C(4)$ & $1.3302(18)$ \\
\hline$C(3)-C(6)$ & $1.5198(18)$ \\
\hline$C(4)-C(5)$ & $1.495(2)$ \\
\hline$C(6)-C(9)$ & $1.526(2)$ \\
\hline $\mathrm{C}(6)-\mathrm{C}(7)$ & $1.527(2)$ \\
\hline$C(6)-C(8)$ & $1.533(2)$ \\
\hline $\mathrm{C}(10)-\mathrm{C}(11)$ & $1.381(2)$ \\
\hline$C(10)-C(15)$ & $1.3966(18)$ \\
\hline $\mathrm{C}(11)-\mathrm{C}(12)$ & $1.380(2)$ \\
\hline$C(12)-C(13)$ & $1.374(3)$ \\
\hline $\mathrm{C}(13)-\mathrm{C}(14)$ & $1.380(3)$ \\
\hline$C(14)-C(15)$ & $1.393(2)$ \\
\hline $\mathrm{C}(15)-\mathrm{N}(1)-\mathrm{C}(16)$ & $113.31(17)$ \\
\hline $\mathrm{C}(15)-\mathrm{N}(1)-\mathrm{C}(17)$ & $114.85(16)$ \\
\hline $\mathrm{C}(16)-\mathrm{N}(1)-\mathrm{C}(17)$ & $112.4(2)$ \\
\hline $\mathrm{O}(1)-\mathrm{S}(1)-\mathrm{C}(10)$ & $105.89(7)$ \\
\hline $\mathrm{O}(1)-\mathrm{S}(1)-\mathrm{C}(1)$ & $106.18(7)$ \\
\hline $\mathrm{C}(10)-\mathrm{S}(1)-\mathrm{C}(1)$ & $96.90(6)$ \\
\hline$C(5)-C(1)-C(2)$ & $105.47(10)$ \\
\hline$C(5)-C(1)-S(1)$ & $114.09(10)$ \\
\hline $\mathrm{C}(2)-\mathrm{C}(1)-\mathrm{S}(1)$ & 107.11(9) \\
\hline $\mathrm{O}(2)-\mathrm{C}(2)-\mathrm{C}(3)$ & $127.83(13)$ \\
\hline $\mathrm{O}(2)-\mathrm{C}(2)-\mathrm{C}(1)$ & $124.20(12)$ \\
\hline$C(3)-C(2)-C(1)$ & $107.96(11)$ \\
\hline $\mathrm{C}(4)-\mathrm{C}(3)-\mathrm{C}(2)$ & $108.13(11)$ \\
\hline
\end{tabular}




$\begin{array}{ll}\mathrm{C}(4)-\mathrm{C}(3)-\mathrm{C}(6) & 128.91(12) \\ \mathrm{C}(2)-\mathrm{C}(3)-\mathrm{C}(6) & 122.97(11) \\ \mathrm{C}(3)-\mathrm{C}(4)-\mathrm{C}(5) & 114.91(12) \\ \mathrm{C}(4)-\mathrm{C}(5)-\mathrm{C}(1) & 103.51(11) \\ \mathrm{C}(3)-\mathrm{C}(6)-\mathrm{C}(9) & 110.20(12) \\ \mathrm{C}(3)-\mathrm{C}(6)-\mathrm{C}(7) & 109.47(12) \\ \mathrm{C}(9)-\mathrm{C}(6)-\mathrm{C}(7) & 109.02(15) \\ \mathrm{C}(3)-\mathrm{C}(6)-\mathrm{C}(8) & 108.76(13) \\ \mathrm{C}(9)-\mathrm{C}(6)-\mathrm{C}(8) & 109.05(15) \\ \mathrm{C}(7)-\mathrm{C}(6)-\mathrm{C}(8) & 110.33(16) \\ \mathrm{C}(11)-\mathrm{C}(10)-\mathrm{C}(15) & 121.87(13) \\ \mathrm{C}(11)-\mathrm{C}(10)-\mathrm{S}(1) & 118.55(10) \\ \mathrm{C}(15)-\mathrm{C}(10)-\mathrm{S}(1) & 119.58(10) \\ \mathrm{C}(12)-\mathrm{C}(11)-\mathrm{C}(10) & 119.61(14) \\ \mathrm{C}(13)-\mathrm{C}(12)-\mathrm{C}(11) & 119.55(16) \\ \mathrm{C}(12)-\mathrm{C}(13)-\mathrm{C}(14) & 120.87(15) \\ \mathrm{C}(13)-\mathrm{C}(14)-\mathrm{C}(15) & 120.90(15) \\ \mathrm{C}(14)-\mathrm{C}(15)-\mathrm{C}(10) & 117.15(13) \\ \mathrm{C}(14)-\mathrm{C}(15)-\mathrm{N}(1) & 124.49(13) \\ \mathrm{C}(10)-\mathrm{C}(15)-\mathrm{N}(1) & 118.34(12)\end{array}$

Symmetry transformations used to generate equivalent atoms: 
Table 4. Anisotropic displacement parameters $\left(\AA^{2} \times 1^{3}\right)$. The anisotropic displacement factor exponent takes the form: $-2 \pi^{2}\left[h^{2} a^{* 2} U^{11}+\ldots+2 h k a^{*} b^{*} U^{12}\right]$

\begin{tabular}{lcccccc}
\hline & $\mathrm{U}^{11}$ & $\mathrm{U}^{22}$ & $\mathrm{U}^{33}$ & $\mathrm{U}^{23}$ & $\mathrm{U}^{13}$ & $\mathrm{U}^{12}$ \\
\hline $\mathrm{N}(1)$ & $74(1)$ & $68(1)$ & $56(1)$ & $-10(1)$ & $2(1)$ & $-13(1)$ \\
$\mathrm{O}(1)$ & $44(1)$ & $122(1)$ & $67(1)$ & $-2(1)$ & $-14(1)$ & $-4(1)$ \\
$\mathrm{O}(2)$ & $122(1)$ & $50(1)$ & $64(1)$ & $0(1)$ & $5(1)$ & $-1(1)$ \\
$\mathrm{S}(1)$ & $46(1)$ & $77(1)$ & $51(1)$ & $0(1)$ & $-4(1)$ & $9(1)$ \\
$\mathrm{C}(1)$ & $43(1)$ & $65(1)$ & $44(1)$ & $-2(1)$ & $-3(1)$ & $-3(1)$ \\
$\mathrm{C}(2)$ & $56(1)$ & $52(1)$ & $50(1)$ & $-1(1)$ & $-2(1)$ & $-4(1)$ \\
$\mathrm{C}(3)$ & $48(1)$ & $52(1)$ & $49(1)$ & $-1(1)$ & $-1(1)$ & $1(1)$ \\
$\mathrm{C}(4)$ & $56(1)$ & $52(1)$ & $58(1)$ & $1(1)$ & $5(1)$ & $5(1)$ \\
$\mathrm{C}(5)$ & $53(1)$ & $65(1)$ & $54(1)$ & $11(1)$ & $2(1)$ & $6(1)$ \\
$\mathrm{C}(6)$ & $71(1)$ & $62(1)$ & $46(1)$ & $-2(1)$ & $-1(1)$ & $7(1)$ \\
$\mathrm{C}(7)$ & $97(1)$ & $77(1)$ & $60(1)$ & $16(1)$ & $14(1)$ & $0(1)$ \\
$\mathrm{C}(8)$ & $91(1)$ & $105(1)$ & $59(1)$ & $-6(1)$ & $-20(1)$ & $23(1)$ \\
$\mathrm{C}(9)$ & $108(2)$ & $76(1)$ & $59(1)$ & $-16(1)$ & $8(1)$ & $5(1)$ \\
$\mathrm{C}(10)$ & $43(1)$ & $63(1)$ & $48(1)$ & $-4(1)$ & $2(1)$ & $6(1)$ \\
$\mathrm{C}(11)$ & $48(1)$ & $73(1)$ & $62(1)$ & $-6(1)$ & $4(1)$ & $-3(1)$ \\
$\mathrm{C}(12)$ & $71(1)$ & $65(1)$ & $71(1)$ & $6(1)$ & $14(1)$ & $1(1)$ \\
$\mathrm{C}(13)$ & $83(1)$ & $79(1)$ & $56(1)$ & $9(1)$ & $1(1)$ & $11(1)$ \\
$\mathrm{C}(14)$ & $72(1)$ & $79(1)$ & $51(1)$ & $-5(1)$ & $-9(1)$ & $3(1)$ \\
$\mathrm{C}(15)$ & $53(1)$ & $60(1)$ & $47(1)$ & $-8(1)$ & $1(1)$ & $5(1)$ \\
$\mathrm{C}(16)$ & $142(2)$ & $62(1)$ & $117(2)$ & $1(1)$ & $10(2)$ & $7(1)$ \\
$\mathrm{C}(17)$ & $86(1)$ & $118(2)$ & $88(1)$ & $-16(1)$ & $1(1)$ & $-41(1)$ \\
& & & & & & \\
\hline & & & & & & \\
& & & & & \\
\hline
\end{tabular}


Table 5. Hydrogen coordinates $\left(x 1^{4}\right)$ and isotropic displacement parameters $\left(\AA^{2} \times 10^{3}\right)$.

\begin{tabular}{|c|c|c|c|c|}
\hline & $\mathrm{x}$ & $\mathrm{y}$ & $\mathrm{z}$ & $\mathrm{U}(\mathrm{eq})$ \\
\hline $\mathrm{H}(1)$ & 2082(16) & 1819(13) & $2490(7)$ & $62(4)$ \\
\hline $\mathrm{H}(4)$ & $1829(15)$ & $-634(15)$ & $1461(7)$ & $69(4)$ \\
\hline $\mathrm{H}(5 \mathrm{~A})$ & $977(15)$ & $-174(14)$ & $2369(6)$ & $65(4)$ \\
\hline $\mathrm{H}(5 \mathrm{~B})$ & $2315(15)$ & $-52(15)$ & $2413(7)$ & $69(5)$ \\
\hline $\mathrm{H}(7 \mathrm{~A})$ & $2553(17)$ & 2603(19) & $677(8)$ & $87(6)$ \\
\hline $\mathrm{H}(7 \mathrm{~B})$ & $2710(20)$ & $1908(17)$ & $126(11)$ & $104(7)$ \\
\hline $\mathrm{H}(7 \mathrm{C})$ & $3410(20)$ & 1563(19) & $639(10)$ & 103(7) \\
\hline $\mathrm{H}(8 \mathrm{~A})$ & $420(20)$ & $2350(20)$ & $684(10)$ & 106(7) \\
\hline $\mathrm{H}(8 \mathrm{~B})$ & $510(20)$ & 1757(19) & $109(11)$ & $112(7)$ \\
\hline $\mathrm{H}(8 \mathrm{C})$ & $-150(20)$ & $1214(18)$ & $636(10)$ & $97(7)$ \\
\hline $\mathrm{H}(9 \mathrm{~A})$ & $1790(20)$ & $125(18)$ & 11(11) & $110(7)$ \\
\hline $\mathrm{H}(9 \mathrm{~B})$ & $1160(20)$ & $-425(18)$ & $505(8)$ & $93(6)$ \\
\hline $\mathrm{H}(9 \mathrm{C})$ & 2539(18) & $-365(18)$ & $529(9)$ & $91(6)$ \\
\hline $\mathrm{H}(11)$ & $-684(16)$ & $60(14)$ & $3189(7)$ & $73(5)$ \\
\hline $\mathrm{H}(12)$ & $-385(18)$ & $-706(17)$ & $4064(8)$ & $86(6)$ \\
\hline $\mathrm{H}(13)$ & $952(17)$ & $88(16)$ & $4651(8)$ & $87(5)$ \\
\hline $\mathrm{H}(14)$ & $1985(18)$ & $1694(16)$ & 4392(9) & $88(6)$ \\
\hline $\mathrm{H}(16 \mathrm{~A})$ & $1080(30)$ & $3950(20)$ & $3955(13)$ & $136(10)$ \\
\hline $\mathrm{H}(16 \mathrm{~B})$ & $1490(30)$ & $4370(30)$ & $3370(13)$ & $140(10)$ \\
\hline $\mathrm{H}(16 \mathrm{C})$ & $320(30)$ & $3680(30)$ & $3351(14)$ & $155(12)$ \\
\hline $\mathrm{H}(17 \mathrm{~A})$ & $3350(20)$ & $3430(20)$ & $3428(11)$ & $114(8)$ \\
\hline $\mathrm{H}(17 \mathrm{~B})$ & $2940(30)$ & $3080(20)$ & $4012(14)$ & 133(9) \\
\hline $\mathrm{H}(17 \mathrm{C})$ & $3390(30)$ & $2230(30)$ & $3623(14)$ & $147(12)$ \\
\hline
\end{tabular}


Table 6. Torsion angles $\left[{ }^{\circ}\right]$.

\begin{tabular}{|c|c|}
\hline $\mathrm{O}(1)-\mathrm{S}(1)-\mathrm{C}(1)-\mathrm{C}(5)$ & $-41.55(11)$ \\
\hline$C(10)-S(1)-C(1)-C(5)$ & $67.25(10)$ \\
\hline $\mathrm{O}(1)-\mathrm{S}(1)-\mathrm{C}(1)-\mathrm{C}(2)$ & $74.77(10)$ \\
\hline $\mathrm{C}(10)-\mathrm{S}(1)-\mathrm{C}(1)-\mathrm{C}(2)$ & $-176.44(9)$ \\
\hline $\mathrm{C}(5)-\mathrm{C}(1)-\mathrm{C}(2)-\mathrm{O}(2)$ & $-178.63(15)$ \\
\hline $\mathrm{S}(1)-\mathrm{C}(1)-\mathrm{C}(2)-\mathrm{O}(2)$ & $59.48(17)$ \\
\hline $\mathrm{C}(5)-\mathrm{C}(1)-\mathrm{C}(2)-\mathrm{C}(3)$ & $0.88(14)$ \\
\hline$S(1)-C(1)-C(2)-C(3)$ & $-121.02(10)$ \\
\hline $\mathrm{O}(2)-\mathrm{C}(2)-\mathrm{C}(3)-\mathrm{C}(4)$ & $177.99(15)$ \\
\hline $\mathrm{C}(1)-\mathrm{C}(2)-\mathrm{C}(3)-\mathrm{C}(4)$ & $-1.49(15)$ \\
\hline $\mathrm{O}(2)-\mathrm{C}(2)-\mathrm{C}(3)-\mathrm{C}(6)$ & $-2.0(2)$ \\
\hline $\mathrm{C}(1)-\mathrm{C}(2)-\mathrm{C}(3)-\mathrm{C}(6)$ & $178.57(11)$ \\
\hline $\mathrm{C}(2)-\mathrm{C}(3)-\mathrm{C}(4)-\mathrm{C}(5)$ & $1.56(16)$ \\
\hline $\mathrm{C}(6)-\mathrm{C}(3)-\mathrm{C}(4)-\mathrm{C}(5)$ & $-178.50(13)$ \\
\hline $\mathrm{C}(3)-\mathrm{C}(4)-\mathrm{C}(5)-\mathrm{C}(1)$ & $-0.97(16)$ \\
\hline $\mathrm{C}(2)-\mathrm{C}(1)-\mathrm{C}(5)-\mathrm{C}(4)$ & $-0.02(14)$ \\
\hline$S(1)-C(1)-C(5)-C(4)$ & $117.24(10)$ \\
\hline $\mathrm{C}(4)-\mathrm{C}(3)-\mathrm{C}(6)-\mathrm{C}(9)$ & $-0.5(2)$ \\
\hline $\mathrm{C}(2)-\mathrm{C}(3)-\mathrm{C}(6)-\mathrm{C}(9)$ & $179.44(14)$ \\
\hline $\mathrm{C}(4)-\mathrm{C}(3)-\mathrm{C}(6)-\mathrm{C}(7)$ & $-120.38(16)$ \\
\hline $\mathrm{C}(2)-\mathrm{C}(3)-\mathrm{C}(6)-\mathrm{C}(7)$ & $59.55(17)$ \\
\hline $\mathrm{C}(4)-\mathrm{C}(3)-\mathrm{C}(6)-\mathrm{C}(8)$ & $119.00(17)$ \\
\hline $\mathrm{C}(2)-\mathrm{C}(3)-\mathrm{C}(6)-\mathrm{C}(8)$ & $-61.06(18)$ \\
\hline $\mathrm{O}(1)-\mathrm{S}(1)-\mathrm{C}(10)-\mathrm{C}(11)$ & $1.68(12)$ \\
\hline $\mathrm{C}(1)-\mathrm{S}(1)-\mathrm{C}(10)-\mathrm{C}(11)$ & $-107.36(11)$ \\
\hline $\mathrm{O}(1)-\mathrm{S}(1)-\mathrm{C}(10)-\mathrm{C}(15)$ & $-177.83(10)$ \\
\hline $\mathrm{C}(1)-\mathrm{S}(1)-\mathrm{C}(10)-\mathrm{C}(15)$ & $73.13(11)$ \\
\hline $\mathrm{C}(15)-\mathrm{C}(10)-\mathrm{C}(11)-\mathrm{C}(12)$ & $-1.1(2)$ \\
\hline$S(1)-C(10)-C(11)-C(12)$ & $179.37(11)$ \\
\hline $\mathrm{C}(10)-\mathrm{C}(11)-\mathrm{C}(12)-\mathrm{C}(13)$ & $-0.5(2)$ \\
\hline $\mathrm{C}(11)-\mathrm{C}(12)-\mathrm{C}(13)-\mathrm{C}(14)$ & $0.8(3)$ \\
\hline $\mathrm{C}(12)-\mathrm{C}(13)-\mathrm{C}(14)-\mathrm{C}(15)$ & $0.6(3)$ \\
\hline $\mathrm{C}(13)-\mathrm{C}(14)-\mathrm{C}(15)-\mathrm{C}(10)$ & $-2.2(2)$ \\
\hline $\mathrm{C}(13)-\mathrm{C}(14)-\mathrm{C}(15)-\mathrm{N}(1)$ & $179.13(14)$ \\
\hline $\mathrm{C}(11)-\mathrm{C}(10)-\mathrm{C}(15)-\mathrm{C}(14)$ & $2.5(2)$ \\
\hline$S(1)-C(10)-C(15)-C(14)$ & $-178.04(11)$ \\
\hline
\end{tabular}



$\mathrm{C}(11)-\mathrm{C}(10)-\mathrm{C}(15)-\mathrm{N}(1)$
$-178.78(12)$
$\mathrm{S}(1)-\mathrm{C}(10)-\mathrm{C}(15)-\mathrm{N}(1)$
$0.72(16)$
$\mathrm{C}(16)-\mathrm{N}(1)-\mathrm{C}(15)-\mathrm{C}(14)$
$-96.5(2)$
$\mathrm{C}(17)-\mathrm{N}(1)-\mathrm{C}(15)-\mathrm{C}(14)$
$34.6(2)$
$\mathrm{C}(16)-\mathrm{N}(1)-\mathrm{C}(15)-\mathrm{C}(10)$
$84.9(2)$
$\mathrm{C}(17)-\mathrm{N}(1)-\mathrm{C}(15)-\mathrm{C}(10)$
$-144.10(16)$

Symmetry transformations used to generate equivalent atoms:

Table 7. Hydrogen bonds [ $\AA^{\AA}$ and ${ }^{\circ}$ ].

\begin{tabular}{lllll}
\hline D-H...A & d(D-H) & d(H...A & d(D...A $)$ & $<($ DHA $)$ \\
\hline
\end{tabular}

